\title{
Investigating the Response of Loop Plasma to Nanoflare Heating Using RADYN Simulations
}

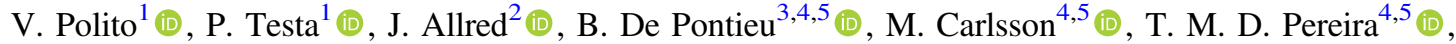 \\ Milan Gošić ${ }^{3,6}$ (iD), and Fabio Reale ${ }^{7}$ (iD \\ ${ }^{1}$ Harvard-Smithsonian Center for Astrophysics, 60 Garden Street, Cambridge, MA 01238, USA \\ ${ }^{2}$ NASA Goddard Space Flight Center, Greenbelt, MD 20771, USA \\ ${ }^{3}$ Lockheed Martin Solar \& Astrophysics Lab, Org. A021S, Bldg. 252, 3251 Hanover Street, Palo Alto, CA 94304, USA \\ ${ }_{5}^{4}$ Rosseland Centre for Solar Physics, University of Oslo, P.O. Box 1029, Blindern, NO-0315 Oslo, Norway \\ ${ }^{5}$ Institute of Theoretical Astrophysics, University of Oslo, P.O. Box 1029, Blindern, NO-0315 Oslo, Norway \\ ${ }^{6}$ Bay Area Environmental Research Institute, Petaluma, CA 94952, USA \\ ${ }^{7}$ Dipartimento di Fisica e Chimica, Università di Palermo and Istituto Nazionale di Astrofisica (INAF)/Osservatorio Astronomico di Palermo, \\ Piazza del Parlamento 1, I-90134 Palermo, Italy \\ Received 2018 January 11; revised 2018 February 26; accepted 2018 March 4; published 2018 April 5
}

\begin{abstract}
We present the results of 1D hydrodynamic simulations of coronal loops that are subject to nanoflares, caused by either in situ thermal heating or nonthermal electron (NTE) beams. The synthesized intensity and Doppler shifts can be directly compared with Interface Region Imaging Spectrograph (IRIS) and Atmospheric Imaging Assembly (AIA) observations of rapid variability in the transition region (TR) of coronal loops, associated with transient coronal heating. We find that NTEs with high enough low-energy cutoff $\left(E_{\mathrm{C}}\right)$ deposit energy in the lower TR and chromosphere, causing blueshifts (up to $\sim 20 \mathrm{~km} \mathrm{~s}^{-1}$ ) in the IRIS Si IV lines, which thermal conduction cannot reproduce. The $E_{\mathrm{C}}$ threshold value for the blueshifts depends on the total energy of the events $(\approx 5 \mathrm{keV}$ for $10^{24} \mathrm{erg}$, up to $15 \mathrm{keV}$ for $10^{25} \mathrm{erg}$ ). The observed footpoint emission intensity and flows, combined with the simulations, can provide constraints on both the energy of the heating event and $E_{\mathrm{C}}$. The response of the loop plasma to nanoflares depends crucially on the electron density: significant Si IV intensity enhancements and flows are observed only for initially low-density loops $\left(<10^{9} \mathrm{~cm}^{-3}\right)$. This provides a possible explanation of the relative scarcity of observations of significant moss variability. While the TR response to single heating episodes can be clearly observed, the predicted coronal emission (AIA $94 \AA$ ) for single strands is below current detectability and can only be observed when several strands are heated closely in time. Finally, we show that the analysis of the IRIS $\mathrm{Mg}$ II chromospheric lines can help further constrain the properties of the heating mechanisms.
\end{abstract}

Key words: line: profiles - Sun: activity - Sun: corona - Sun: transition region

\section{Introduction}

Understanding the details of the physical mechanism(s) responsible for the heating of the solar and stellar coronae to temperatures exceeding several million kelvin still represents one of the most challenging problems in astrophysics. Although significant progress in theoretical modeling and major observational advancements have been achieved in recent years, we still lack a definitive solution to the coronal heating problem (see, e.g., reviews by Klimchuk 2006, 2015; Reale 2014; Testa et al. 2015). Among different competing mechanisms, smallscale impulsive nanoflare events $\left(E \approx 10^{24}-10^{25} \mathrm{erg}\right)$, associated with magnetic reconnection in the corona, are a popular candidate process for converting magnetic energy into the thermal energy required to heat the active region (AR) plasma (e.g., Parker 1988; Priest et al. 2002). We note that coronal heating models alternative to the nanoflare scenario, such as heating by Alfvén waves and associated with spicules, have also been suggested (e.g., De Pontieu et al. 2011, 2017; van Ballegooijen et al. 2011).

Several authors have focused on constraining the properties of the coronal heating models by comparing the plasma parameters derived from spectroscopic and imaging observations (i.e., flows, line broadening, emission measure, line intensity ratio) with the predictions of numerical simulations (e.g., Warren et al. 2008; Reale et al. 2009; Testa et al. 2011, 2016; Winebarger et al. 2011). However, observing the direct signatures of energy release in the corona is complicated by many factors, including the low density of the plasma and the presence of nonequilibrium effects, and therefore only limited constraints can often be obtained for the models.

On the other hand, the observation of the upper transition region (TR; $T \sim 0.08-0.15 \mathrm{MK}$ ) footpoints of hot $(T \gtrsim 3 \mathrm{MK})$ coronal loops (i.e., "moss") provides highly sensitive diagnostics, because of the rapid variation of the physical conditions of the local plasma in response to the heating. The low-lying coronal plasma was first obtained in the X-rays with the Normal Incidence X-Ray Telescope (NIXT; e.g., Peres et al. 1994) and then identified as upper TR moss by the Transition Region and Coronal Explorer (TRACE; Handy et al. 1999) in narrow EUV bands centered around $\approx 1 \mathrm{MK}$ (e.g., Berger et al. 1999; De Pontieu et al. 1999; Fletcher \& De Pontieu 1999; Martens et al. 2000). Some of the studies using TRACE images, as well as spectroscopic observations from instruments such as the Hinode/EUV Imaging Spectrometer (EIS; Culhane et al. 2007), showed low variability of the moss region, on timescales of the order of several minutes, which had often been interpreted as an indication of quasi-steady heating of the coronal loops (e.g., Antiochos et al. 2003; Brooks \& Warren 2009; Tripathi et al. 2010; Warren et al. 2010), or as due to other phenomena (e.g., De Pontieu et al. 2003, who established that variability on timescales of 3-5 minutes is caused by chromospheric jets obscuring upper TR material). In 2012, the launch of the High-resolution Coronal Imager 
(Hi-C; Kobayashi et al. 2014), on board a sounding rocket flight, provided a very different and much clearer view of the moss dynamics, thanks to its unprecedented spatial (0". 3-0." 4) and temporal $(5.5 \mathrm{~s})$ resolution. Hi-C observations revealed highly variable TR moss emission on timescales down to $15 \mathrm{~s}$ associated with coronal activity, supporting the impulsive nanoflare heating scenario (e.g., Testa et al. 2013). However, Hi-C lacked spectroscopic measurements, which are crucial to provide detailed information about the physical conditions of the plasma and therefore key observables to compare with the predictions of the models.

Since its launch in 2013, the Interface Region Imaging Spectrometer (IRIS; De Pontieu et al. 2014) has provided simultaneous observations of chromosphere and lower TR spectra and images at very high spatial $(0 ! 33)$, spectral ( $\approx 3 \mathrm{~km} \mathrm{~s}^{-1}$ ), and temporal (up to $1-2 \mathrm{~s}$ ) resolutions. IRIS has greatly improved on the past spectroscopic instruments, which were limited by lower spatial resolution and the lack of coaligned context imaging observations. In particular, recent work by Testa et al. (2014, hereafter T14) has demonstrated that IRIS observations of the TR footpoint variability combined with advanced numerical simulations can provide tight constraints on the nanoflare models. More specifically, T14 showed that the blueshifts observed in the TR Si IV line at $1402.77 \AA\left(T \approx 10^{4.9} \mathrm{~K}\right)$ with IRIS could not be reproduced by models assuming heating by thermal conduction only, but were consistent with electron beam heating, highlighting for the first time the possible importance of nonthermal electrons in the heating of nonflaring ARs. T14 also combined images from the Atmospheric Imaging Assembly (AIA; Lemen et al. 2012) on board the Solar Dynamic Observatory (SDO; Pesnell et al. 2012) to precisely locate the TR footpoints of the hot coronal loops. Judge et al. (2017) recently analyzed additional magnetic data for the observations of T14 and found that at one location the ribbon appears possibly magnetically disconnected from the corona. In light of this intriguing possibility, Judge et al. (2017) discuss alternative heating scenarios (e.g., chromospheric reconnection) for that one ribbon location, although, as already discussed by T14, these alternative mechanisms appear unlikely for the vast majority of the observed TR brightenings.

The numerical investigation carried out by T14 was performed with the RADYN code (Carlsson \& Stein 1992, 1995, 1997; Allred et al. 2015) using a limited set of model input parameters aimed at reproducing the particular observation under study. However, IRIS observations indicate a large variety of properties for the observed footpoint brightenings, which show lifetimes of 10-30 s, display a broad range of Doppler shifts in the TR and chromospheric lines (from blue to red or no shifts), and appear connected to coronal loops with a range of different lengths $(\sim 10-100 \mathrm{Mm})$, as observed by AIA (P. Testa et al. 2018, in preparation). To understand and interpret the variety of TR observations, in this work we carry out a detailed analysis of nanoflare simulations, exploring a much wider region in the parameter space compared with the analysis of T14. In particular, we explore the atmospheric response to different nanoflare heating models as a function of parameters such as the electron low-energy cutoff, initial loop temperature, length, and total nanoflare energy. We also compare in detail the predictions of nanoflare models assuming heating by either electron beams or in situ heating of the corona. Here we do not aim to forward model a specific observation, but we wish to provide a grid of models, with a wide range of parameter values, that can be used as a guide for the interpretation of IRIS observations of footpoint variability associated with coronal heating. At the same time, we aim to investigate the applicability and limitations of the IRIS and SDO/AIA diagnostics of nanoflare heating.

The paper is organized as follows: In Section 2 we describe the RADYN code and the set of nanoflare simulations we performed. Section 3 presents the evolution of atmospheric response for one set of simulations, whereas Section 4 examines the forward modeling of the IRIS and AIA optically thin plasma observables. In Section 5 we briefly present the results of synthetic chromospheric $\mathrm{Mg}$ II emission observed by IRIS for our different nanoflare models. The main results for the whole parameter space are then discussed in Section 6. Finally, in Section 7 we summarize our main findings and draw our conclusions.

\section{The Numerical Investigation}

We perform simulations of nanoflare-heated loops using the RADYN numerical code. RADYN solves the equation of charge conservation and the level population rate equations on a single 1D magnetic strand in the field-aligned direction, rooted in the photosphere and stretching out to include the chromosphere, TR, and corona. A key advantage of RADYN compared to other 1D hydrodynamic codes is the ability to model the important elements for the chromospheric energy balance $(\mathrm{H}, \mathrm{He}, \mathrm{Ca}$ II) in non-local thermodynamic equilibrium (non-LTE). The chromospheric losses for these elements are calculated in detail using non-LTE radiative transfer, whereas other atomic species are included as background continuum opacity sources (in LTE) using the Uppsala opacity package (Gustafsson 1973). The radiative loss functions for optically thin lines are calculated using the CHIANTI 7.1 (Dere et al. 1997; Landi et al. 2013) atomic database assuming ionization and thermal equilibrium. RADYN uses an adaptive grid (Dorfi \& Drury 1987) with a fixed number of grid cells, the size and location of which can vary to allow shocks and steep gradients in the atmosphere to be resolved.

In this work, we use the state-of-the-art version of RADYN described in Allred et al. (2015), with the following two main modifications: (1) we extended the number of grid points (from 191 to 300), to allow for a sufficient resolution of even the steepest gradients arising from the release of energy in the loop; and (2) we added a more realistic atmospheric structure for the loops, including a plage-like chromosphere following the work of Carlsson et al. (2015).

As discussed in the introduction, there is observational evidence of high variability in the TR and chromosphere associated with coronal heating, which, combined with modeling, can provide important diagnostics of the heating properties. Here we aim to use RADYN to explore two likely scenarios for coronal nanoflare models: (1) transport by nonthermal electrons (accelerated in the corona) that deposit their energy in different layers of the atmosphere, depending on their energy distribution properties and the physical conditions of the plasma in the loop; a power-law distribution for the electron beams is usually assumed in RADYN, with userspecified values of low-energy cutoff $\left(E_{\mathrm{C}} ; \mathrm{keV}\right)$, total energy $\left(E\right.$; erg), energy flux $\left(F\right.$; erg $\left.\mathrm{cm}^{-2} \mathrm{~s}^{-1}\right)$, and spectral index $(\delta)$; and (2) local heating of the corona, with subsequent transport of the energy to the lower atmosphere by thermal conduction. To this aim, we have explored an extensive parameter space for 
our simulations guided by previous modeling results (T14) and recent observations (P. Testa et al. 2018, in preparation), including different heating and loop properties. In particular, as summarized and discussed below:

1. Heating models:

(a) Electron beam (hereafter EB) model, with $E=6 \times$ $10^{24} \mathrm{erg}, \delta=7$, and $E_{\mathrm{C}}=5,10$, and $15 \mathrm{keV}$;

(b) In situ heating of the corona at the loop top (thermal conduction model, hereafter TC), with total energy of $6 \times 10^{24} \mathrm{erg}$.

2. Half-loop lengths $L / 2: 15,50$, and $100 \mathrm{Mm}$.

3. Initial loop-top temperatures $\left(T_{\mathrm{LT}}\right): 1,3$, and $5 \mathrm{MK}$.

The total energy that we choose to deposit in a single loop is representative of the typical values assumed for nanoflares $\left(10^{24}-10^{25} \mathrm{erg}\right.$; e.g., Parker 1988), and it reproduced TR brightenings similar to the IRIS observations in T14, whereas the choice of $\delta=7$ for the electron energy distribution is motivated by the observational trend of increasing spectral index with decreasing flare class (e.g., Hannah et al. 2011). A steep electron distribution means that more electrons have energy close to $E_{\mathrm{C}}$ and are therefore closer to iso-energetic beams, allowing us to study separately the effects of energy deposition by electrons of varying energies. We choose three values of low-energy cutoff $E_{\mathrm{C}}$, resulting in beams that are dominated by low- $(5 \mathrm{keV})$, intermediate- $(10 \mathrm{keV})$, and highenergy $(15 \mathrm{keV})$ electrons.

Our initial loops are obtained assuming initial hydrostatic equilibrium for the plasma, and therefore loops at different temperatures will have different densities. In particular, hotter loops will also be denser. We chose a range of different initial temperature and density conditions that aim to reproduce a large variety of observed AR loops at different heating stages. In particular, low-temperature and longer loops have a lower density and are representative of empty strands before any heating has taken place, whereas hotter (and denser) loops represent strands that have been previously heated and filled with plasma.

All the simulations have been performed for a loop with a cross-sectional area $A$ of $5 \times 10^{14} \mathrm{~cm}^{2}$ (corresponding to a diameter of $\approx 250 \mathrm{~km}$ ). Note that the loop cross-section area does not enter directly in the model, but is only a normalization parameter. We assume the cross section to be constant in our calculations. However, Mikić et al. (2013) showed that the assumption of a nonuniform cross section might affect the evolution of the loops and, in particular, enhance the coronal emission, as we also briefly discuss in Section 4.2.

Further, we assume that the loop strand is heated constantly for $10 \mathrm{~s}$, which is consistent with the lifetimes of short-lived brightenings typically observed in TR moss, as discussed in the introduction. Given the values of loop cross section and heating duration above, the electron beam energy flux $(F)$ will be of the order of $1.2 \times 10^{9} \mathrm{erg} \mathrm{s}^{-1} \mathrm{~cm}^{-2}$ for our EB model runs. Finally, we also discuss the effect of varying the total energy input (from $10^{24}$ to $10^{25} \mathrm{erg}$ ) for one set of loop length and initial apex temperature.

As mentioned above, we adopt an initial atmosphere for the 1D loops that is based on the work by Carlsson et al. (2015), aimed at reproducing $\mathrm{Mg}$ II line profiles that are closer to the observed ones in the plage. Figure 1 shows the initial atmospheric structure for the $15 \mathrm{Mm}$ loop with initial apex temperatures of 1 and $3 \mathrm{MK}$ that we adopted in this work. Note

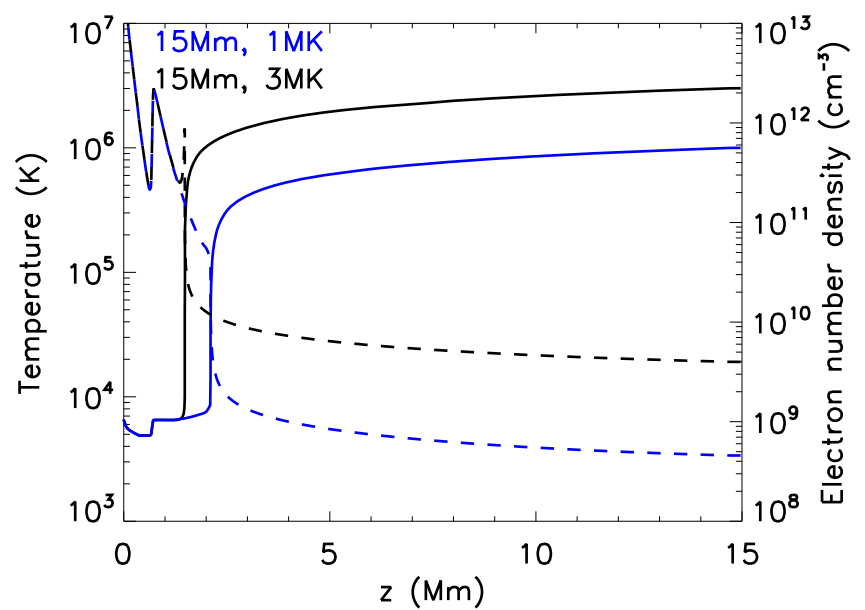

Figure 1. Temperature (solid lines) and electron number density (dotted lines) for the loops with plage-like atmospheres and initial apex temperature of $1 \mathrm{MK}$ (blue) and 3 MK (black).

the presence of a hot chromosphere with a steep temperature rise.

Over 40 numerical simulations were performed, and our key findings are presented as follows: In Section 3 we discuss the results of atmospheric response of the loop to the nanoflare heating for the model run with a $15 \mathrm{Mm}$ loop and initial $T_{\mathrm{LT}}$ of 1 and $3 \mathrm{MK}$, and in Sections 4 and 5 we describe the model predictions of specific IRIS and AIA observables and associated diagnostics for these sets of simulations. In Section 6, the results for the full parameter space, including other loop lengths (50 and $100 \mathrm{Mm}$ ), temperatures (5 MK), and different total energy input ( $E=10^{24}-10^{25} \mathrm{erg}$ ), will be briefly discussed, mainly focusing on the differences with the model predictions presented in Sections 3-5. Finally, Section 7 summarizes the key conclusions of our numerical investigation.

\section{Atmospheric Response for the $15 \mathrm{Mm}$ Loop}

In this section, we describe the evolution of the atmospheric variables as a function of time for the $15 \mathrm{Mm}$ half-loop length models, assuming electron beam (Section 3.1) and in situ (Section 3.2) heating.

\subsection{Electron Beam Heating}

Figures 2 and 3 compare the evolution of the atmospheric variables in the EB heating models as a function of different $E_{\mathrm{C}}$ for the $15 \mathrm{Mm}$ half-loop with initial loop-top temperature $T_{\mathrm{LT}}=1$ and $3 \mathrm{MK}$. These two loops have coronal density $N_{e, \mathrm{LT}}=\approx 10^{8.7}$ and $10^{9.6} \mathrm{~cm}^{-3}$, respectively. Both figures show (from top to bottom) the plasma temperature, electron density, velocity, and beam heating deposition rate per mass $H$ as a function of time for the first $20 \mathrm{~s}$ into the simulations with a $1 \mathrm{~s}$ interval. $H$ is given by the ratio of the particle beam heating rate $Q_{\text {beam }}$ (see Equation (18) of Allred et al. 2015) and the plasma density. Note that only the first $10 \mathrm{~s}$ of the simulation are shown in the heating rate plots, as the heating is switched off after this time. We focus here on the discussion of the first $20 \mathrm{~s}$, because we are interested in comparing in detail the atmospheric response for different models during the heating and the early relaxation phase after the heating is switched off. We point out that the evolution timescales will be different for loops with different temperatures and densities. Nevertheless, 

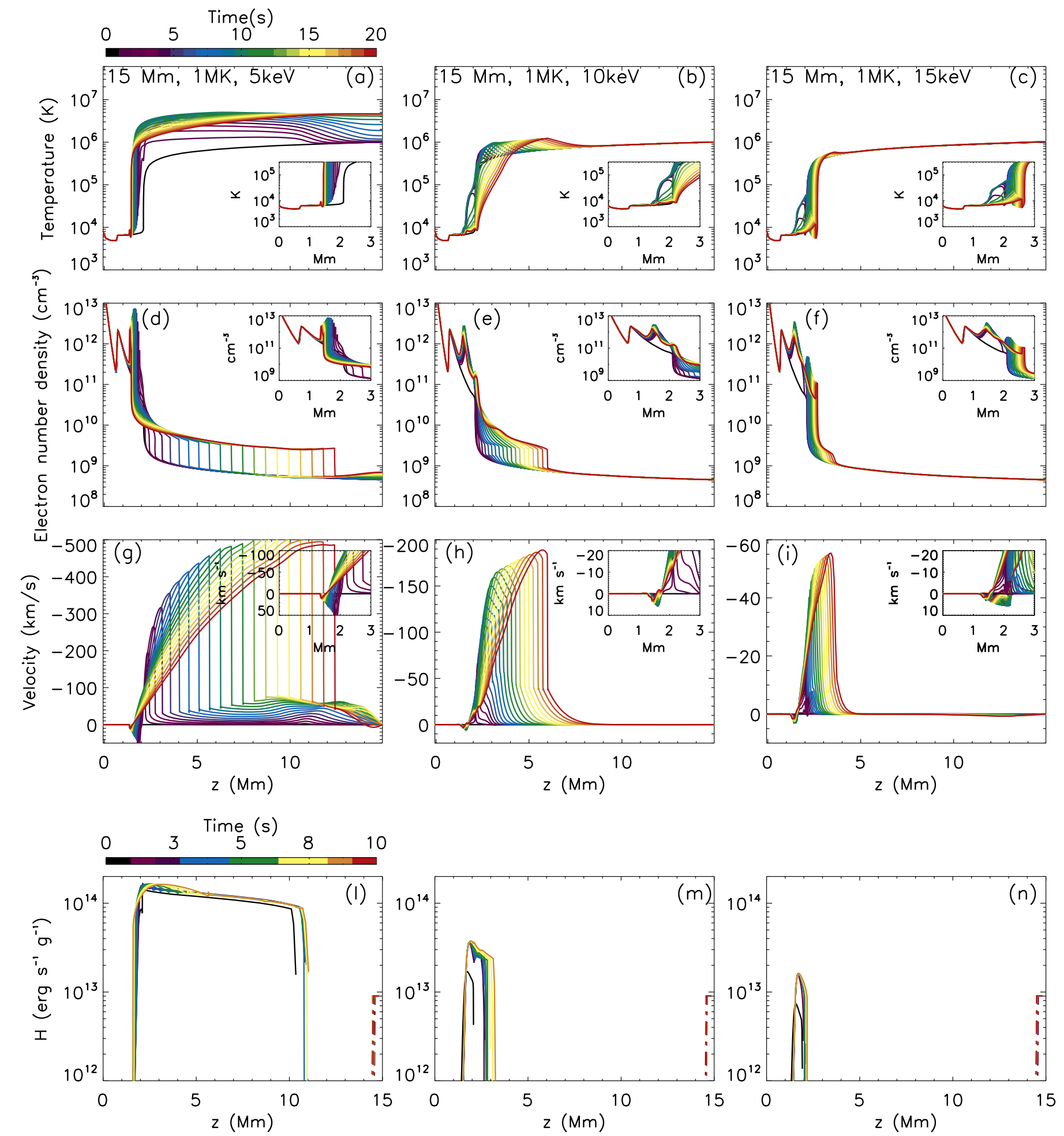

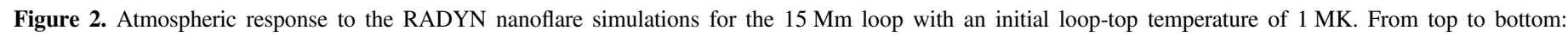

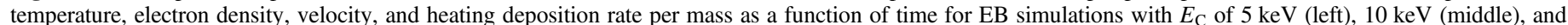

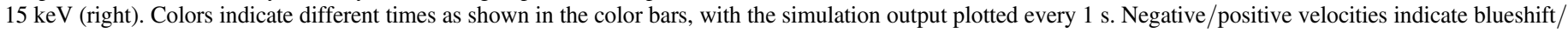

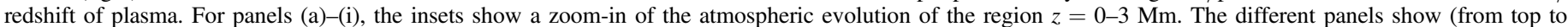

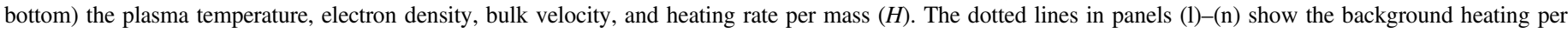

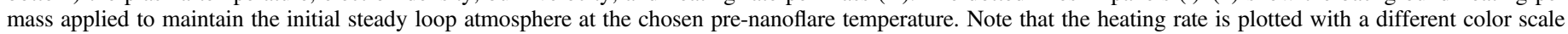
(with relative color bar), as the heating is switched off after $10 \mathrm{~s}$ in the simulations.

we focus our attention on a small timescale because, as mentioned earlier, the lifetimes of the observed TR moss brightenings are of the order of 10-30 s (Testa et al. 2013, 2014; P. Testa et al. 2018, in preparation).
The different columns in Figures 2 and 3 show the results for EB simulations with (from left to right) $E_{\mathrm{C}}=5,10$, and $15 \mathrm{keV}$. Note that negative (positive) velocities mean upflows (downflows) of plasma. The insets in Figure 2 show a zoom-in 


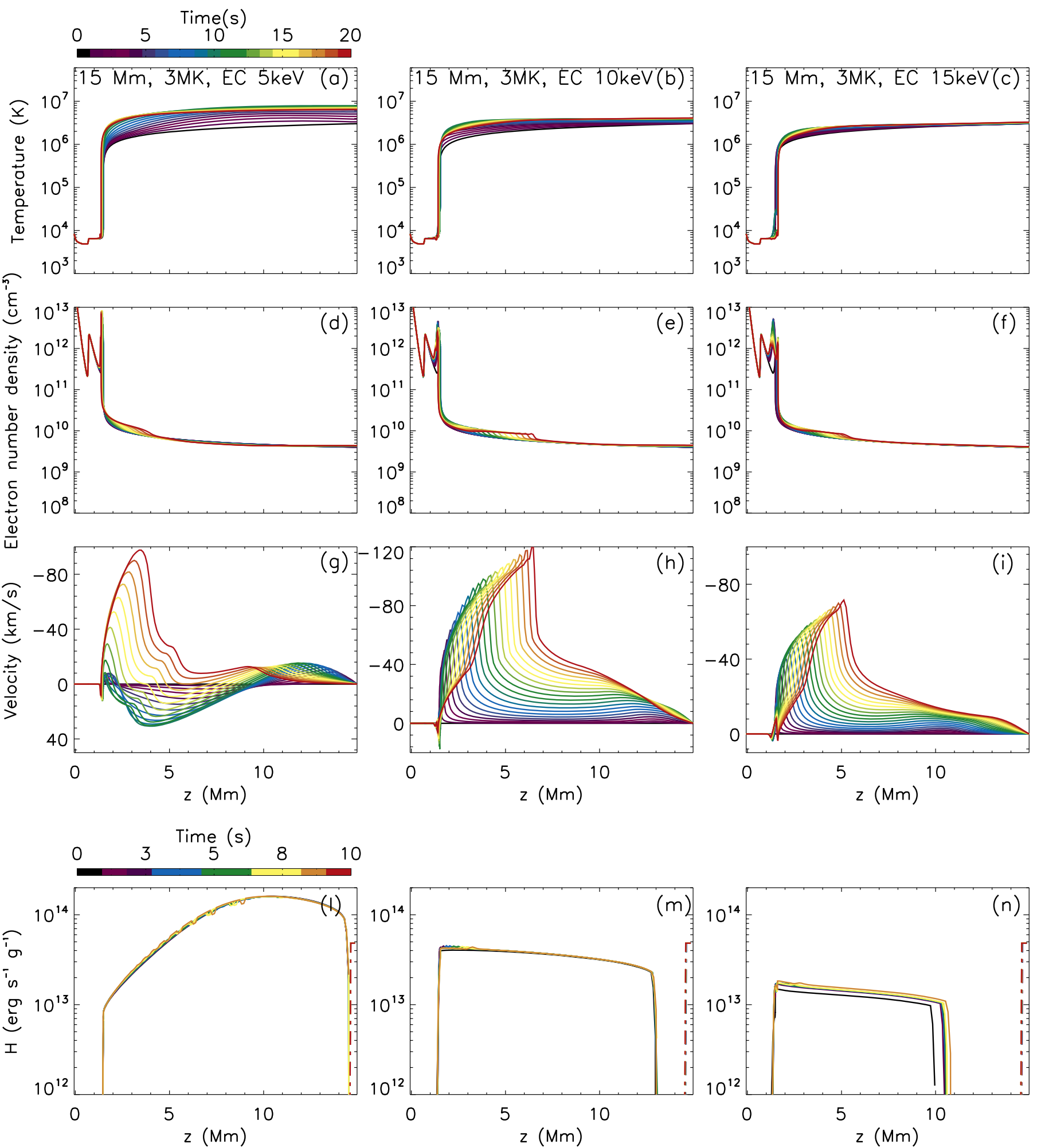

Figure 3. Atmospheric response to the RADYN nanoflare simulations for the $15 \mathrm{Mm}$ loop with an initial loop-top temperature of $3 \mathrm{MK}$. See the caption of Figure 3 for an explanation of the different panels.

of the dynamics of the chromospheric evolution between $z=1$ and $3 \mathrm{Mm}$. Comparing these different sets of simulations provides important information about the energy deposition in the atmosphere as a function of the low-energy cutoff $E_{\mathrm{C}}$. We discuss the results from the runs with $T_{\mathrm{LT}}=1$ and $3 \mathrm{MK}$ separately in the following two sections.

\subsubsection{Mm, 1 MK Loop-top Temperature}

The low-energy electrons $\left(E_{c}=5 \mathrm{keV}\right.$; left panels of Figure 2) mainly deposit their energy in situ in the corona and TR, causing an increase of temperature (panel (a)) and density (panel (d)) in the corona up to around 1 order of magnitude in the first $10 \mathrm{~s}$ and causing the position of the TR to 
recede back toward higher column masses (or lower depths). The TR adjusts in column mass until the resulting increased radiative losses in the TR can balance the incoming thermal conductive flux from the coronal energy deposition. The electrons lose all their energy and are completely stopped at heights between $z \approx 1.5$ and $2 \mathrm{Mm}$. The temperature increase due to the beam heating causes an increase of pressure and therefore very large upward motions (up to $-500 \mathrm{~km} \mathrm{~s}^{-1}$ ) of high-temperature plasma (above $10^{6} \mathrm{~K}$ ), increasing with atmospheric height. At the same time, the pressure gradient drives a significant downflow of TR plasma $\left(\approx 50 \mathrm{~km} \mathrm{~s}^{-1}\right)$ toward the chromosphere. After the heating is switched off, the atmosphere starts cooling off, initially from the lower regions where the denser plasma radiates more efficiently.

Electron distributions with $E_{\mathrm{C}}=10$ and $15 \mathrm{keV}$ (middle and right panels of Figure 2) contain more energetic electrons that are able to propagate through the corona without losing much energy and penetrate deeper into the atmosphere. As a result, there is no (for $E_{\mathrm{C}}=15 \mathrm{keV}$ ) or little (for $E_{\mathrm{C}}=10 \mathrm{keV}$ ) direct heating of the corona compared to the $E_{\mathrm{C}}=5 \mathrm{keV}$ run, but most of the energy is directly deposited in the TR and chromosphere. This can be best seen in the bottom panels ((m)-(n)), showing the evolution of energy deposition per mass as a function of height. In the first few seconds, the beam energy deposition is maximum between 1.5 and $2 \mathrm{Mm}$. Hydrogen and helium ionize quickly, and the radiative losses can no longer balance the large heating deposited there, resulting in a large increase of temperature localized in that region. The overpressure then drives an upflow of plasma from the low atmosphere toward the corona, similarly to the $E_{\mathrm{C}}=5 \mathrm{keV}$ case discussed above.

We note that the upflow velocities are lower in the simulations with $E_{\mathrm{C}}$ of 10 and $15 \mathrm{keV}$ than the one with $5 \mathrm{keV}$. The higher-energy electrons can in fact penetrate deeper into the chromosphere, where the plasma is denser. This means that plasma is harder to accelerate, but also that it will radiate the energy away more efficiently, resulting in a lower pressure increase. Both effects contribute to produce lower upflow velocities for the 10 and $15 \mathrm{keV}$ compared to the $5 \mathrm{keV}$ case. The upflows of plasma in the 10 and $15 \mathrm{keV}$ simulations also cause the TR to be pushed out toward greater heights.

In all three cases, the loop density increases over time because of the strong upflows of plasma toward the corona (chromospheric evaporation), and consequently the loops are filled with high-temperature plasma. As a result, the electrons start depositing more energy at increasingly higher atmospheric layers, as can be seen from the heating deposition rate plots (panels (1)-(n)).

We note that the low-energy electrons are more effective at heating the corona and driving evaporation of high-temperature plasma, in agreement with what was found earlier by Peres et al. (1987) and more recently by T14 and by Reep et al. (2015) in their study of chromospheric evaporation as a function of different electron properties for flare-size events.

\subsection{2. $15 \mathrm{Mm}, 3 \mathrm{MK}$ Loop-top Temperature}

Figure 3 shows the atmospheric response to the EB heating models for the $15 \mathrm{Mm}$ half-loop with initial $T_{\mathrm{LT}}=3 \mathrm{MK}$. As mentioned before and also shown in Figure 1, the initial looptop density is almost one order of magnitude higher than in the 1 MK loop. Because of the higher density, more beam energy is deposited in the corona compared to the $T_{\mathrm{LT}}=1 \mathrm{MK}$ loop, regardless of the electron energy. In the $E_{\mathrm{C}}=5 \mathrm{keV}$ run (left panels), a significant fraction of beam energy is released in the corona (see panel (1)), causing an increase of temperature and pressure driving an upflow and downflow of plasma away from the site of maximum energy release $(\approx 10 \mathrm{Mm})$ in the first $\approx 15 \mathrm{~s}$. Not all the electrons are stopped in the corona, but some of them are able to reach the TR and deposit their energy directly at an atmospheric height of $z \approx 1.5 \mathrm{Mm}$. At that location, the combination of energy deposition and a thermal conduction front from the corona causes a pressure gradient and resulting upflowing plasma toward the corona and a downflowing plasma at low speeds in the TR from around $10 \mathrm{~s}$ onward.

In the $E_{\mathrm{C}}=10$ and $15 \mathrm{keV}$ simulations (middle and right panels), the beam energy is initially deposited in the TR (as shown in panels (m) and (n)), causing upflows of plasma from that region toward the corona. As the loop starts being filled with high-temperature plasma, the electrons get stopped at progressively greater heights, and as a consequence the evaporation front travels upward along the loop (see panels (h)-(i)).

The results above indicate that, for the same heating model, the flows and density/temperature increase in the lower atmosphere are much lower for a denser and hotter loop, where the energy is dissipated more efficiently in the coronal part of the loop. In addition, the response of the atmosphere to the heating by low-energy electrons or thermal conduction (see below Section 3.2) is much slower (after $\sim 10 \mathrm{~s}$ ), because of the higher loop density, which means that the mass is harder to accelerate.

\subsection{Thermal Conduction Heating}

In this section we describe the evolution of the atmospheric variables in the RADYN nanoflare simulations assuming in situ heating of the corona and subsequent transport through thermal conduction, without the presence of accelerated electrons. As mentioned in Section 2, the total energy deposited locally in the corona is the same as the energy released by the accelerated electrons for the EB simulations.

Figure 4 shows the results of the TC simulations for the $15 \mathrm{Mm}$ half-loop with $1 \mathrm{MK}$ (left panels) and $3 \mathrm{MK}$ (right panels) initial loop-top temperatures. The figure shows (from top to bottom) the evolution of the plasma temperature, density, bulk velocity, and heating rate per mass (which we call $H_{\mathrm{TC}}$ ).

We have explored the possibility of releasing the energy over different lengths in the corona. We note that, unless the heating is strongly concentrated at the footpoints (e.g., Müller et al. 2003, 2004; Testa et al. 2005), the details of the spatial distribution of the heating are not crucially affecting the plasma evolution, because the thermal conduction is efficient in redistributing the energy in the corona. We show here the results for a simulation in which heating is distributed over the uppermost $\approx 9 \mathrm{Mm}$ of the loop. In the cooler and less dense $T_{\mathrm{LT}}=1 \mathrm{MK}$ loop, the heat causes a quick increase of the coronal temperature to about $20 \mathrm{MK}$ in the first few seconds into the simulation. The conduction front then reaches and starts heating the TR after $\approx 3 \mathrm{~s}$, causing an increase of pressure driving very large upflows $\left(\sim-500 \mathrm{~km} \mathrm{~s}^{-1}\right)$ of hot plasma toward the corona (panel (e)). Figure 4(e) also shows systematic positive velocities in the TR, i.e., a downflow, which corresponds to redshifted TR lines (e.g., Si IV lines observed by IRIS; see Figure 5), as also discussed by T14. 


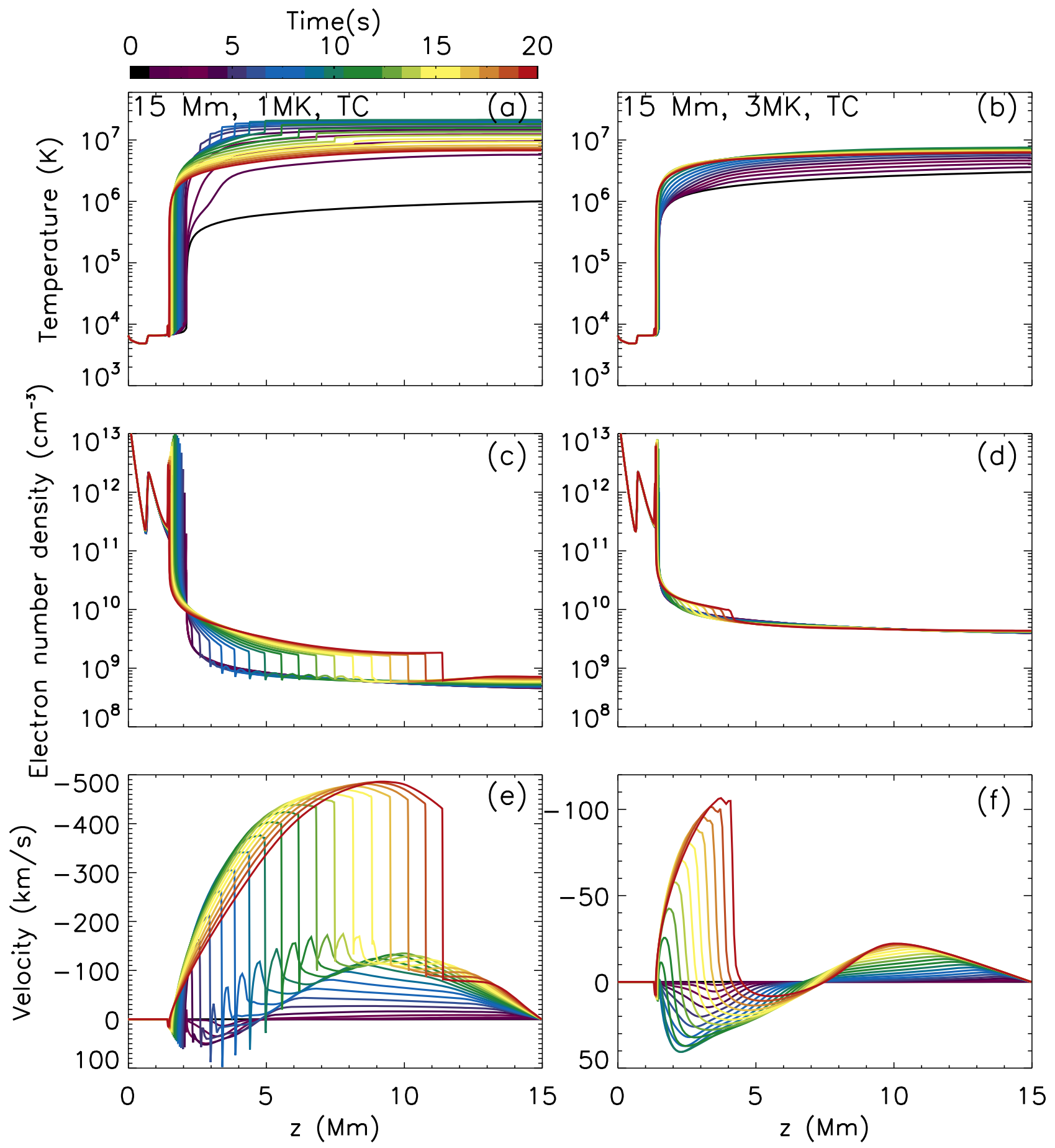

Figure 4. Atmospheric response to the RADYN nanoflare thermal conduction simulations for the $15 \mathrm{Mm}$ loop with initial loop-top temperature of $1 \mathrm{MK}$ (left panels) and $3 \mathrm{MK}$ (right panels). The different panels show (from top to bottom) the plasma temperature, electron density, bulk velocity, and heating rate per mass $\left(H_{\mathrm{TC}}\right)$. The dotted lines in panels $(\mathrm{g})$ and $(\mathrm{h})$ show the background heating per mass applied to maintain the initial steady loop atmosphere at the chosen pre-nanoflare temperature.

In the $T_{\mathrm{LT}}=3 \mathrm{MK}$ loop, the corona has a larger heat capacity and reaches a lower maximum temperature of $\approx 7.5 \mathrm{MK}$. The heating is less effective, and plasma flows are delayed, slower, and less significant here. Moreover, as soon as the heating ends $(t \sim 10 \mathrm{~s})$, the denser plasma abruptly begins to cool down and a pressure dip forms in the low corona, driving an initial downflow (Reale 2016), which then turns into an upflow when the chromospheric plasma takes over.

The atmospheric response to the heating in the TC models shows very similar results to the simulations with EB heating and $E_{\mathrm{C}}=5 \mathrm{keV}$ discussed in Section 3.1. This is also the case for the forward modeling of the plasma emission, as will be shown in Section 4.

\section{Forward Modeling of the Optically Thin and Chromospheric Emission}

We forward model the predicted emission from our simulations in the IRIS Si IV spectral line and in the $94 \AA$ filter of AIA. The Si IV line $\left(T \approx 10^{4.9} \mathrm{~K}\right)$ is the brightest optically 


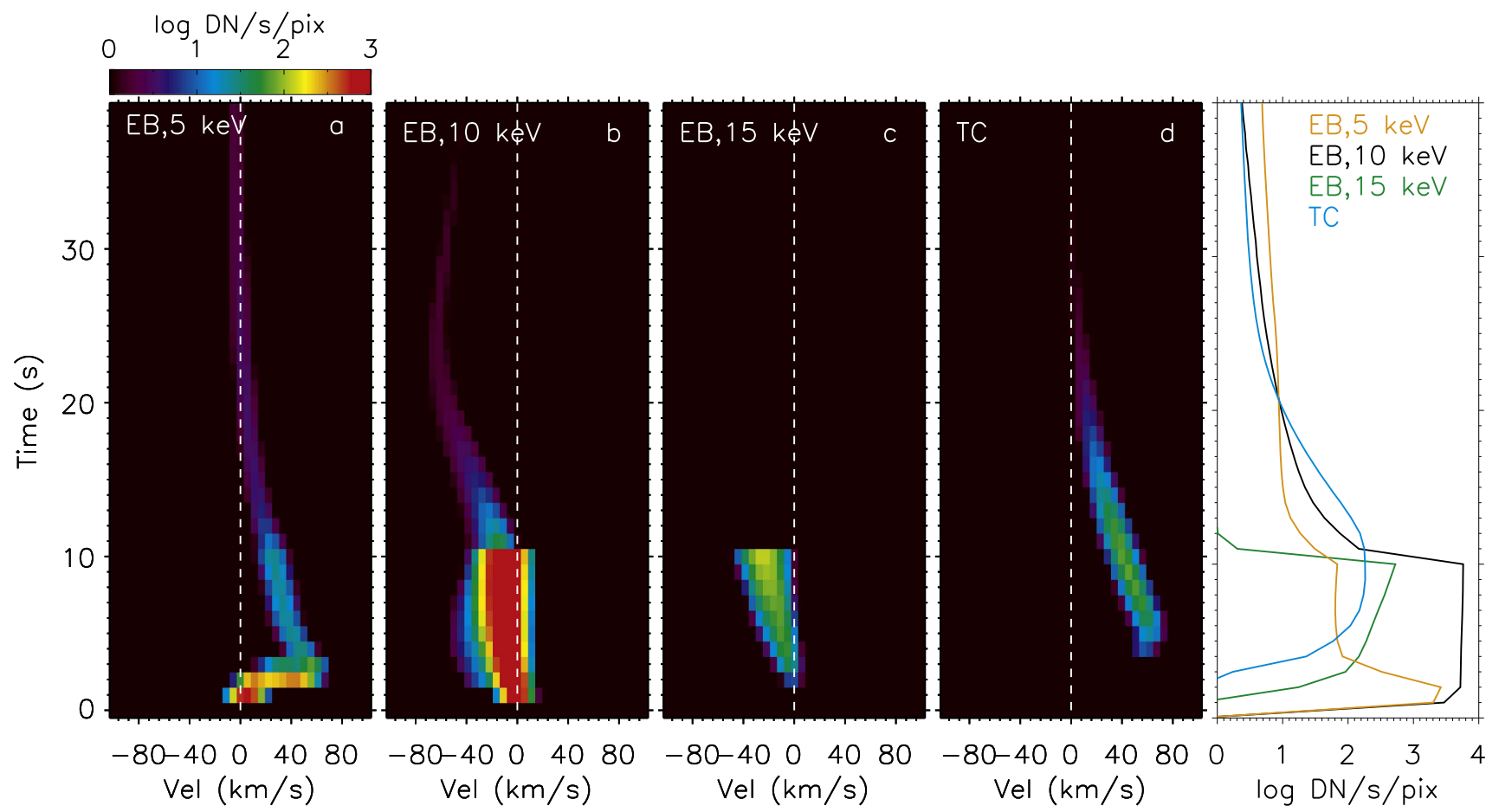

Figure 5. Synthetic Si IV spectra (panels (a)-(d)) and light curves (panel (e)) as a function of time as forward modeled from the RADYN nanoflare simulations assuming $10 \mathrm{~s}$ heating by electron beams with $E_{\mathrm{C}}$ of (a) $5 \mathrm{keV}$, (b) $10 \mathrm{keV}$, and (c) $15 \mathrm{keV}$ and (d) thermal conduction only for the $15 \mathrm{Mm}$ loop with initial loop-top temperature of $1 \mathrm{MK}$. Negative (positive) velocities indicate blueshifts (redshifts).

thin TR line observed by IRIS and provides a powerful diagnostic of moss emission at the footpoints of the nanoflare loops. On the other hand, the AIA $94 \AA$ channel is dominated, in the core of ARs, by plasma at around $\gtrsim 4 \mathrm{MK}$ from hot coronal loops (e.g., Testa \& Reale 2012).

The synthetic emission from the two instruments was calculated using the following formula:

$$
\begin{aligned}
& I\left(\lambda, T(z, t), N_{\mathrm{e}}(z, t)\right)=G\left(\lambda, T(z, t), N_{\mathrm{e}}(z, t)\right) \\
& \quad \cdot R_{\mathrm{I}} \cdot N_{\mathrm{e}}(z, t) \cdot N_{\mathrm{H}}(z, t) \cdot d z(z, t),
\end{aligned}
$$

where I $\left(\lambda, T(z, t), N_{\mathrm{e}}(z, t)\right)$ is the intensity of the lines expressed in $\mathrm{DN} \mathrm{s}^{-1}$ pixel $^{-1}$ as a function of the position $z$ along the loop and time $t . \mathrm{G}\left(\lambda, T(z, t), N_{\mathrm{e}}(z, t)\right)$ represents the contribution function (photons $\mathrm{cm}^{3} \mathrm{sr}^{-1} \mathrm{~s}^{-1}$ ) calculated using atomic data from CHIANTI v.8 (Dere et al. 1997; Del Zanna et al. 2015), assuming coronal abundances (Feldman et al. 1992) and standard SolarSoft (Freeland \& Handy 1998) CHIANTI routines: emiss_calc for the forward modeling of the Si IV line and isothermal for the AIA $94 \AA$, following the method described in the Appendix of Del Zanna et al. (2011). $R_{\mathrm{I}}$ is the instrument response function (units: DN photons ${ }^{-1} \mathrm{sr} \mathrm{cm}^{2}$ pixel $^{-1}$ ), given by the product of the effective area, plate scale, and gain of the telescopes. For AIA, we use a plate scale of $8.46 \times$ $10^{-2}$ sr pixel $^{-1}$, while the effective area and gain were obtained using the aia_get_response $(/ d n)$ routine. For IRIS, we use $4 \mathrm{DN}$ photon $^{-1}$ for the far-UV (FUV) spectrograph channel and the effective areas given by the iris_get_response routine. Finally, $N_{\mathrm{e}}$, $N_{\mathrm{H}}$, and $d z$ represent the electron and hydrogen number density and grid size, respectively, which are given by the RADYN models and vary as a function of $z$ and $t$.

\subsection{IRIS Si IV Emission}

Using Equation (1), we have synthesized the evolution of the Si IV spectra for our different nanoflare models. We assume that the loop is observed from above, and thus the Si IV emission from the loop footpoints is spatially integrated along the line of sight and is observed in one IRIS pixel. The synthetic spectra are obtained including an instrumental width for the FUV channel of $31.8 \mathrm{~m} \AA .{ }^{8}$ Figures 5 and 7 show the evolution of the Si IV spectra as a function of time (y-axis) and Doppler shift velocity ( $x$-axis) for the first $40 \mathrm{~s}$ into the nanoflare simulations. Negative (positive) velocity indicates blueshifts (redshifts), consistent with Figures 2-4. The intensity of the line (in logarithmic scale) is shown in reversed colors as indicated by the color bar on the top of panel (a). From left to right, panels (a)-(d) present the Si IV synthetic spectra for the EB simulations with $E_{\mathrm{C}}=5,10$, and $15 \mathrm{keV}$ and the TC simulation, respectively. Panel (e) shows the Si IV light curves, obtained by integrating the emission over the spectral line, for the four simulations listed above.

In Figure 5, both the intensity images and the light curves show a sudden increase of intensity of the Si IV line over the first few seconds in response to the heating. The Si IV width also increases by a factor of two or more (differently for different heating models) as a result of the superposition of different line components (shifted and at rest) along the line of sight. In particular, the intensity rise is more dramatic in the EB simulations, where the emission increases up to 3-4 orders of magnitude over the first $10 \mathrm{~s}$, before dropping dramatically to its pre-heating value. In order to better understand this sudden drop in intensity, in Figure 6 we show the electron number

8 iris.lmsal.com/itn26/codes.html 


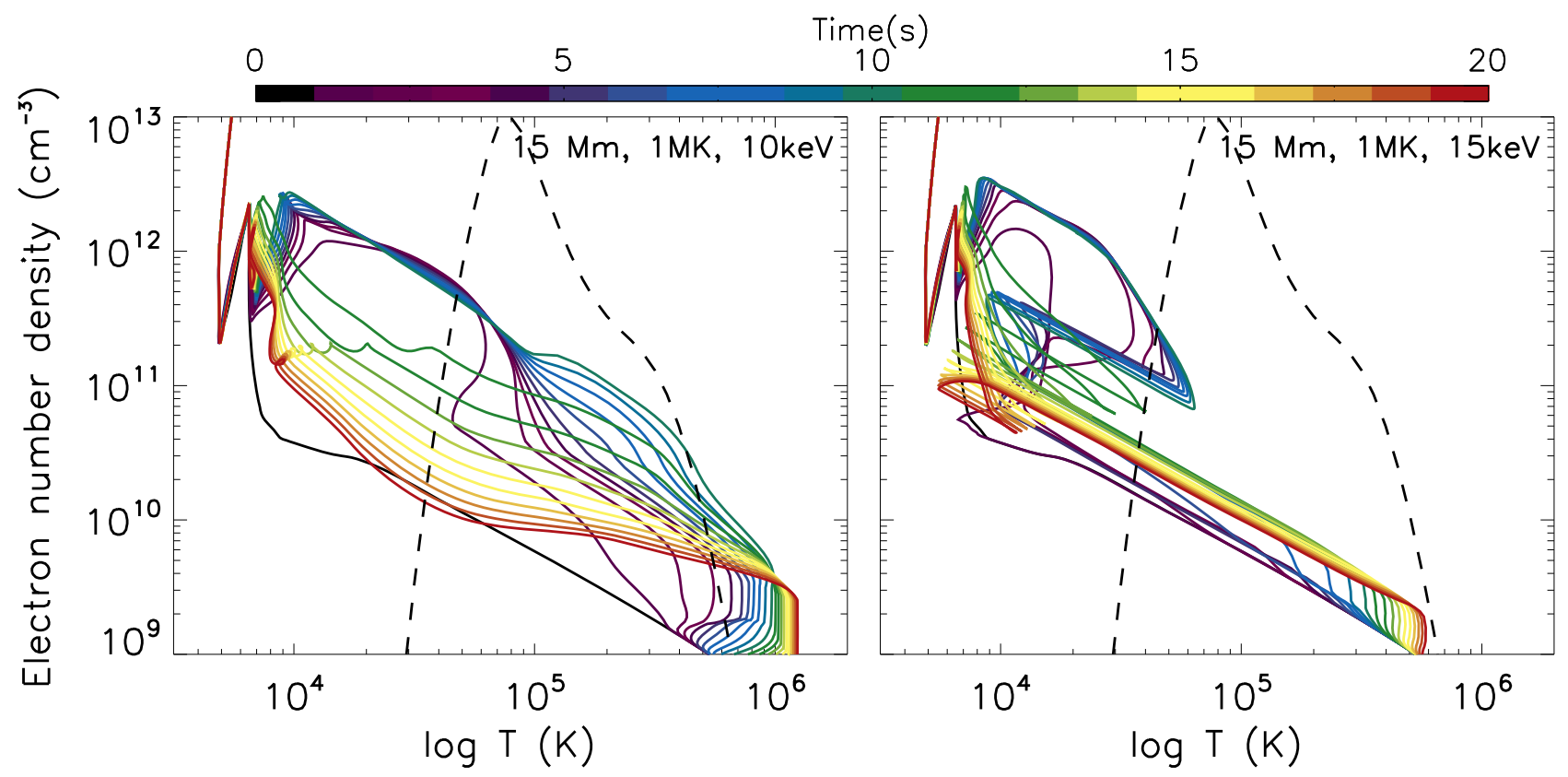

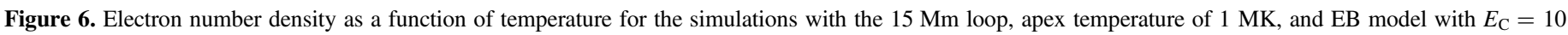

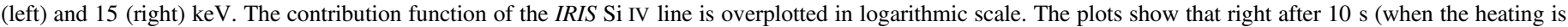
switched off) the density drops significantly within the temperature range of formation of the ion.

density as a function of temperature for the simulations with the $15 \mathrm{Mm}$ loop, apex temperature of $1 \mathrm{MK}$, and $\mathrm{EB}$ model with $E_{\mathrm{C}}=10$ (left panel) and 15 (right panel) $\mathrm{keV}$. The dotted curve overlaid on the figure represents the contribution function of the Si IV line, which has been normalized to arbitrary units. This figure shows that after $10 \mathrm{~s}$ (when the heating is switched off) the electron density drops significantly within the temperature range of formation of the Si IV line, justifying the rapid decrease in intensity in the spectra in Figure 5. This sudden variation in the Si IV intensity is compatible with the observations of short-lived (10-30 s) TR brightenings in AR heating events (e.g., Testa et al. 2013; T14; P. Testa et al. 2018, in preparation), as discussed in the introduction. In addition, the maximum Si IV intensity increase and line broadening are caused with the $10 \mathrm{keV}$ electrons, which deposit a large fraction of their energy at a depth where most of the Si IV emission is formed. We note that both the line intensity and width (in addition to the Doppler shift) represent crucial parameters that can help distinguish between different heating models.

In the $E_{\mathrm{C}}=5 \mathrm{keV}$ simulation (panel (a)), we observe a steep increase of intensity and a large Si IV redshift up to around $50 \mathrm{~km} \mathrm{~s}^{-1}$ in the first $\approx 3 \mathrm{~s}$. As explained in Section 3.1.1, at this time the electrons deposit a significant fraction of their energy in the TR, causing a large downflow of plasma as a consequence of the pressure gradient. A large Si IV redshift is also observed in the TC simulation (panel (d)) after around $\approx 3 \mathrm{~s}$, in agreement with what we described in Section 3.2.

In the $3 \mathrm{MK}$ loop simulations (Figure 7), the intensity increase of the Si IV line is much lower than in the $1 \mathrm{MK}$ case described above (note that we used the same intensity color scale for both Figures 5 and 7). The $3 \mathrm{MK}$ loop is in fact significantly denser than the $1 \mathrm{MK}$ loop, and as a result, the electron energy is dissipated more efficiently before reaching the TR and chromosphere, as also discussed in Section 3.1.2. The EB simulations with $E_{\mathrm{C}}=10$ and $15 \mathrm{keV}$ (panels (b) and (c)) show a moderate blueshift (up to $30 \mathrm{~km} \mathrm{~s}^{-1}$ ) of the Si IV line profile in the first $10 \mathrm{~s}$, while in the $E_{\mathrm{C}}=5 \mathrm{keV}$ and
TC cases (panels (a) and (d)) the line appears to be slightly redshifted $\left(\approx 20 \mathrm{~km} \mathrm{~s}^{-1}\right)$ after $10 \mathrm{~s}$. As discussed in Sections 3.1.2 and 3.2, in these cases the TR is in fact heated by the thermal conduction front from the corona only after $\approx 10 \mathrm{~s}$ into the simulations.

Figures 5 and 7 show that the evolution of the Si IV spectra is very sensitive to both the details of the heating model and the initial atmosphere, as also shown and discussed in T14. The results for the other nanoflare models with half-loop lengths of 50 and $100 \mathrm{Mm}, T_{\mathrm{LT}}=5 \mathrm{MK}$, and different total energy will be discussed in Section 6.

\subsection{Coronal Emission}

In contrast to the TR footpoint emission, the modeling of the coronal emission from the nanoflare loops observed by AIA requires some additional considerations:

(1) To take into account the fact that the loop cross section $\left(A=5 \times 10^{14} \mathrm{~cm}^{2}\right)$ is much smaller than the AIA pixel area $\left(A_{\text {AIA_pix }} \approx 1.89 \times 10^{15} \mathrm{~cm}^{2}\right)$, we multiply Equation (1) by an additional factor, given by the ratio of these two values $\left(A / A_{\text {AIA_pix }} \approx 0.26\right)$. (2) We note that the AIA $94 \AA$ passband is sensitive to both hot emission (from Fe XVIII; $\approx 7 \mathrm{MK}$ ) and cooler emission (e.g., O’Dwyer et al. 2010; Martínez-Sykora et al. 2011; Del Zanna 2012; Testa et al. 2012; Boerner et al. 2014). To isolate the emission from the hot core AR loops, we only model the emission above $3 \mathrm{MK}$ due to Fe XVIII. This approach allows us to directly compare the results of our simulations with both imaging observations with AIA, where the Fe XVIII emission is often isolated by removing the cooler contaminating component from the $94 \AA$ channel, and spectroscopic data from instruments such as Hinode/EIS, observing emission lines formed at similar temperatures to the Fe XVIII (see, e.g., Reale et al. 2011; Testa \& Reale 2012; Del Zanna 2013; Ugarte-Urra \& Warren 2014). (3) We calculate the total emission per AIA pixel by simply dividing the total emission integrated along the coronal part of the loop 


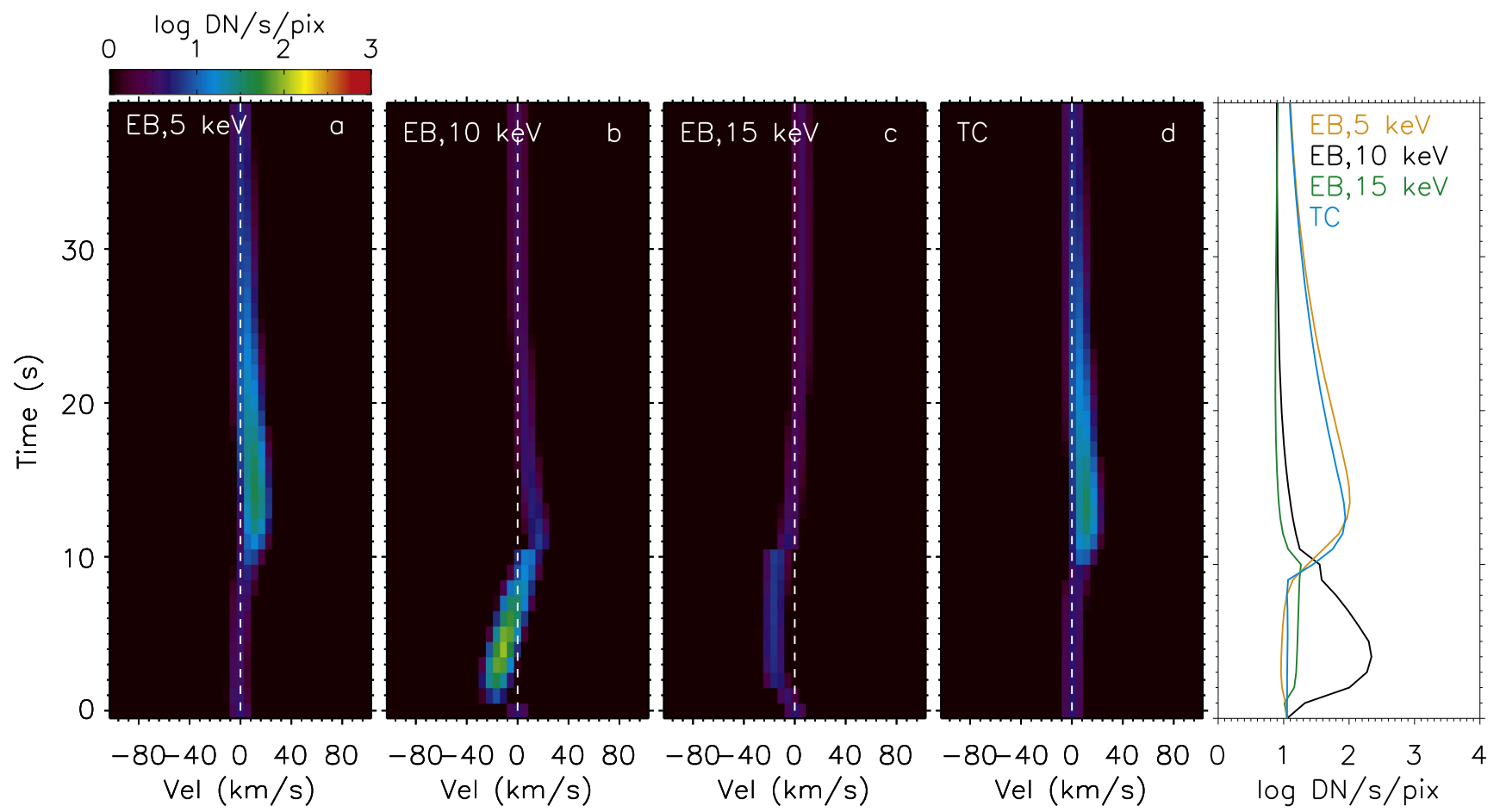

Figure 7. Synthetic Si IV spectra and light curves for the $15 \mathrm{Mm}$ loop with initial loop-top temperature of $3 \mathrm{MK}$. Negative (positive) velocities indicate blueshifts (redshifts). See caption of Figure 5 for more details.
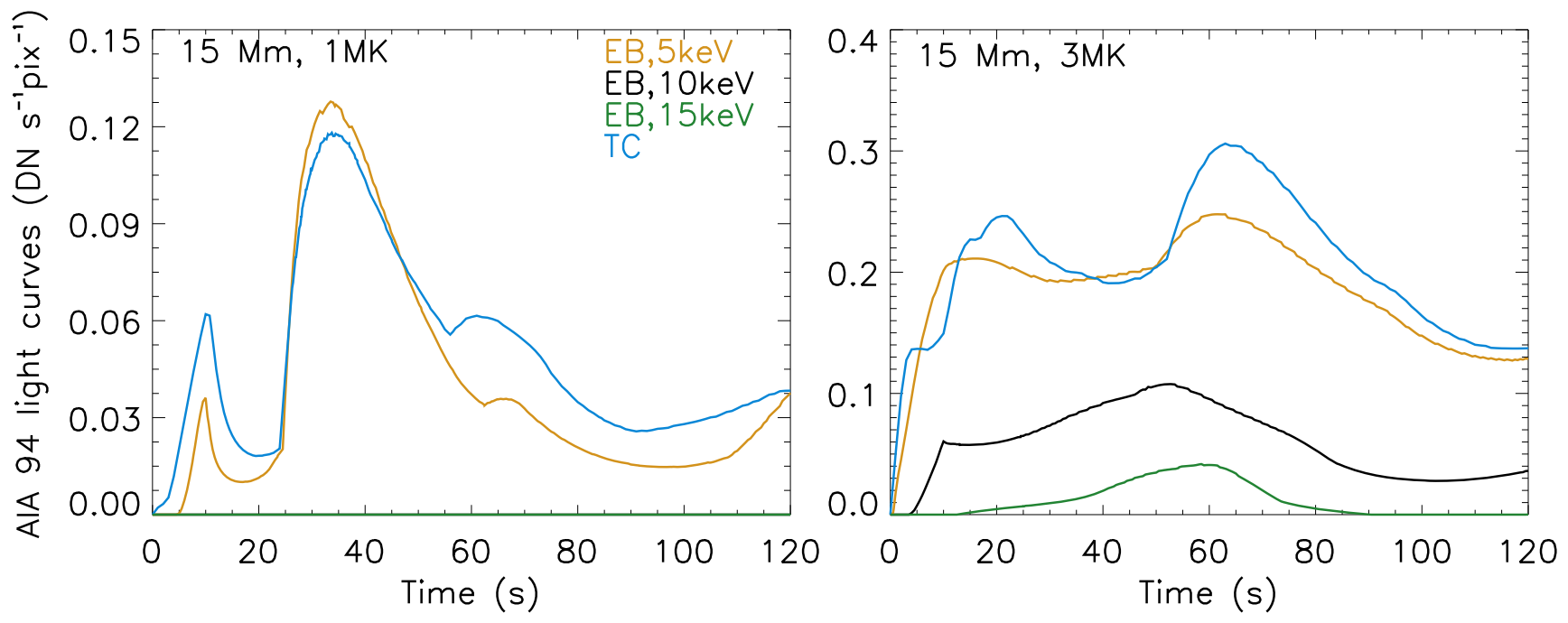

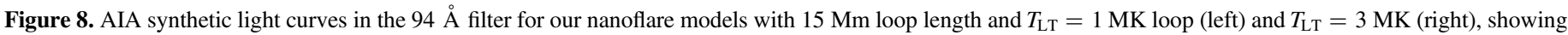

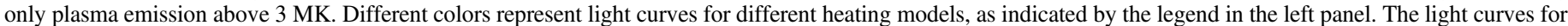

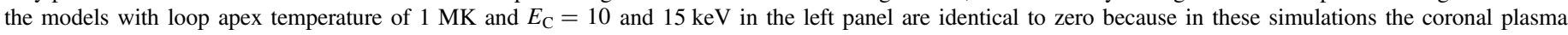
temperature does not reach $3 \mathrm{MK}$.

(where the temperature is above $3 \mathrm{MK}$ ) by the corresponding number of AIA pixels along that length. While this assumption does not take into account any geometrical effects (which vary from case to case depending on the line of sight of the observations and on the inclination of the loops), it provides a lower limit of the emission that can be observed in one AIA pixel.

Figure 8 shows the AIA synthetic light curves ( $\mathrm{DN} \mathrm{s}^{-1}$ pixel $^{-1}$ ) in the $94 \AA$ filter for the $15 \mathrm{Mm}$ half-loop length with $T_{\mathrm{LT}}=1 \mathrm{MK}$ loop (left panel) and $T_{\mathrm{LT}}=3 \mathrm{MK}$ (right panel) and different heating models, for the first $120 \mathrm{~s}$ into the simulations. The figure indicates that the light curves for the $T_{\mathrm{LT}}=3 \mathrm{MK}$ loop are higher (by a factor of at least 2) than those for the $T_{\mathrm{LT}}=1 \mathrm{MK}$ loop, regardless of the properties of the heating model, due to the significantly higher initial temperature and density of the initial loop.

In addition, as discussed in the previous sections, the electrons deposit more energy in the corona in the case of the denser $3 \mathrm{MK}$ loop than in the almost empty $1 \mathrm{MK}$ loop. We also note that the light curves for the models with loop apex temperature of $1 \mathrm{MK}$ and $E_{\mathrm{C}}=10$ and $15 \mathrm{keV}$ (left panel) are identical to zero because in these simulations the coronal plasma temperature does not reach $3 \mathrm{MK}$, which we have used as the threshold value for calculating the "hot" $94 \AA$ emission. 

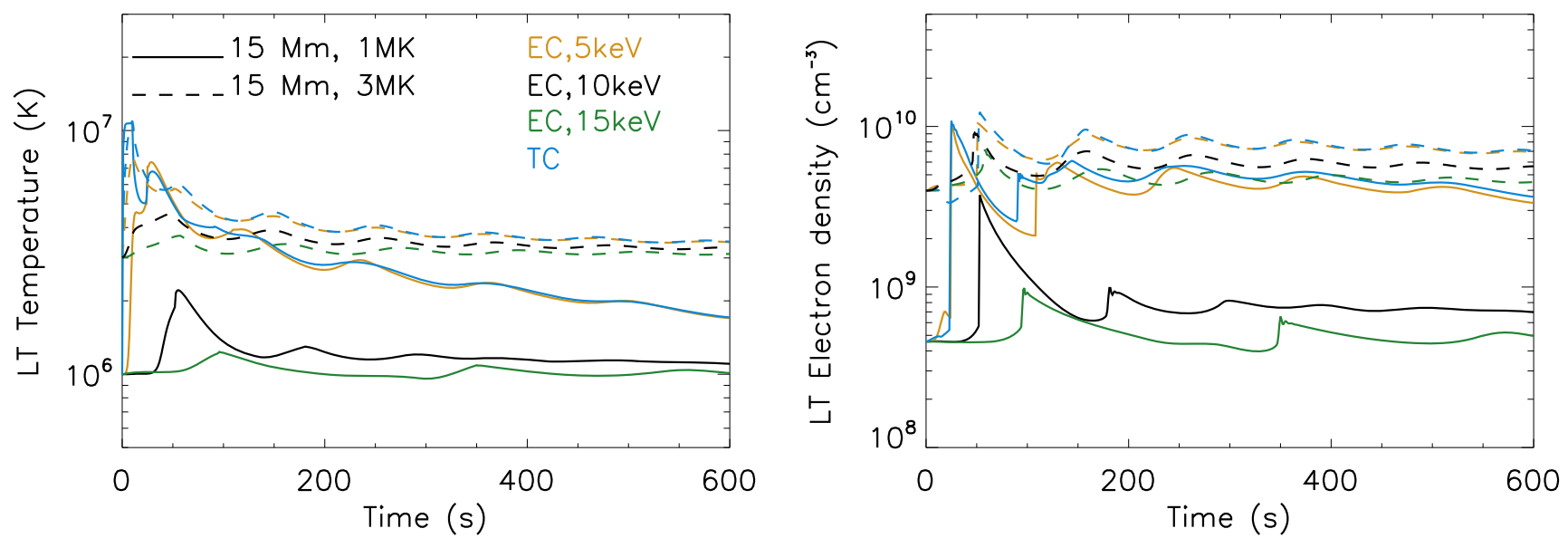

Figure 9. Apex temperature (left) and electron number density (right) for the $15 \mathrm{Mm}$ loop run as a function of time for the first $600 \mathrm{~s}$ into the simulations. Different colors represent different heating models, whereas different line styles (solid and dashed) indicate different initial temperature for the loops, as indicated in the legend in the left panel.

Figure 8 also shows that, for both loop temperatures, the simulations with $\mathrm{TC}$ only and $\mathrm{EB}$ heating with $E_{\mathrm{C}}=5 \mathrm{keV}$ produce higher $94 \AA$ emission than the EB simulations with $E_{\mathrm{C}}=10$ and $15 \mathrm{keV}$. As discussed in Section 3, this is due to the fact that in the first two models most of the heating is deposited directly in the corona, causing a larger increase of the coronal temperature.

The temporal evolution of the light curves shows a first peak between 10 and $20 \mathrm{~s}$, when the heating is deposited, and a second, more intense peak between 40 and $60 \mathrm{~s}$ into the simulations following the chromospheric evaporation, due to the loop being filled with hot upflowing plasma from the footpoints. After this time, the light curves first decrease (in the first $200 \mathrm{~s}$ ) and then remain constant over time, in a similar fashion to the temperature and density evolution at the loop top. This can be best seen in Figure 9, showing the apex temperature (left) and density (right) as a function of time for the different models. The curves show an oscillating pattern, which is the result of the evaporating plasma being reflected at the upper boundary of the loop, mimicking the effect of a symmetrical loop footpoint.

The AIA light curves in Figure 8 show that the peak intensity for a $15 \mathrm{Mm}$ loop is observed to be within $100 \mathrm{~s}$ after the heating has taken place, while in observations the peak of the AIA $94 \AA$ emission for the hot loops is often observed on longer timescales (from few to tens of minutes; P. Testa et al. 2018, in preparation). This is not surprising and could indicate that multiple impulsive heating events over time might be needed to reproduce the observed light curves, i.e., a "nanoflare train" (e.g., Klimchuk 2006; Bradshaw et al. 2012; Reep et al. 2013). In addition, longer loops also produce AIA emission for a longer time; for instance, in our simulations with $100 \mathrm{Mm}$ and apex temperature of $3 \mathrm{MK}$, the peak intensity in the $94 \AA$ filter occurs around $400 \mathrm{~s}$, as discussed in Section 6.1.

The time delay between the TR and coronal emission can in principle provide important information about the mode of energy transport and the loop conditions. For instance, for a denser loop and $5 \mathrm{keV}$ and TC simulations, the delay between the peak of the TR and coronal emission is smaller than in the case of a less dense loop, as can be seen by comparing Figures 5 and 8. However, in some cases such delays may be too short to be observed by AIA, which has a temporal cadence of $\approx 12 \mathrm{~s}$.
Further, we point out that the count rates shown in Figure 8 for our single nanoflare loop models, although representing a lower limit, are a couple of orders of magnitude lower than the typically observed values of $\sim 20-30 \mathrm{DN} \mathrm{s}^{-1} \mathrm{pixel}^{-1}$, as observed for the coronal loops overlying the footpoint brightening observed by Testa et al. (2013) and T14 (see also Testa \& Reale 2012; Ugarte-Urra \& Warren 2014). We can thus provide a rough estimate of approximately 100-200 loop strands, which would be needed for reproducing the typical AR core observations of the coronal plasma. This estimate is consistent with previous calculations of, e.g., Peter et al. (2013). This might also explain the delayed observed intensity peak: the real light curve would be an envelope of pulsed light curves slightly time-shifted one from the other, as the heat pulses are likely not synchronous from one strand to the other (e.g., Reale et al. 2012; Tajfirouze et al. 2016). We also note that the events showing moss variability associated with brightening of hot coronal loops typically involve a large number of footpoints. Many loops appear to be heated at slightly different times, and while the footpoint brightenings are well separated owing to their short duration, the coronal emission has significantly longer timescales, and many loop strands can contribute to the coronal emission observed in a pixel at a given time. For instance, for the events discussed in T14 and Testa et al. (2013), the footpoint brightenings cover an overall area of $\approx 10^{16}-10^{17} \mathrm{~cm}^{2}$ (i.e., up to several hundred times our assumed loop cross section). Therefore, the overall observed hot emission (peaking around $25 \mathrm{DN} \mathrm{s}^{-1}$ pixel $^{-1}$ in the $94 \AA$ AIA band) is compatible with the values predicted by the simulations (Figure 8).

Furthermore, higher total energies for the heating events could also produce higher coronal emission (see Section 6.3). Finally, a likely scenario is also that of a hybrid model in which a portion of the energy of the heating episode is released locally in the corona, even in events in which significant nonthermal particles are present (and might dominate the chromospheric and TR heating). Another possible explanation for the low coronal emission in our single nanoflare models might be related to the choice of uniform cross section for the loops in our simulations. Mikić et al. (2013) showed in fact that loops with nonconstant cross sections are more likely to develop thermal nonequilibrium, resulting in a significantly enhanced coronal emission. 
The fact that several loop strands (of the order of 100-200) might be needed to reproduce the AIA observations suggests that the cumulative hard X-ray emission emitted by the accelerated electrons in those strands might be strong enough to be detected by current instruments. T14 (Supplementary text S3) calculated the predicted X-ray emission observed by RHESSI for a single nanoflare loop (with the same total energy as in our work) to be around 0.1 counts for $10 \mathrm{~s}$ integration time and per detector. The nanoflare events are likely not occurring simultaneously, and considering that 100-200 events might occur during an integration time of $30 \mathrm{~s}$, that would result in about 30-60 total counts detector ${ }^{-1}$ or $1-2$ counts $s^{-1}$ detector $^{-1}$. Therefore, we expect most of these events to be below or close to the RHESSI sensitivity limit (of the order of 2-7 counts $\mathrm{s}^{-1}$ detector $^{-1}$ for a 30 s integration time; Saint-Hilaire et al. 2009), though some might be detected by RHESSI. The The Nuclear Spectroscopic Telescope Array (NUSTAR; Harrison et al. 2013) has a better sensitivity and lower background than RHESSI and might be able to observe more of these nanoflare-size events.

\section{IRIS Mg II Chromospheric Emission}

We synthesized the spectra of the chromospheric Mg II $h$ and $k$ lines at 2803.53 and $2796.35 \AA$ observed by IRIS using the RH1.5D radiative transfer code (Uitenbroek 2001; Pereira \& Uitenbroek 2015). The RH code calculates the Mg II lines in partial frequency redistribution (PRD). In PRD, the frequencies of the radiation absorbed and re-emitted by an atom are correlated. We use a 21-level plus continuum model for the $\mathrm{Mg}$ II atom. We note that the RADYN calculations do not include PRD, but rather assume complete frequency redistribution (CRD). Under the CRD assumption, the radiation scattering is completely incoherent. While this is not appropriate for line synthesis (for which we use RH including PRD), as shown by Leenaarts et al. (2013a), it is a reasonable approximation for the energetics in the RADYN simulations.

We use snapshots of the atmosphere evolution calculated by RADYN every $1 \mathrm{~s}$ as input to the RH1.5D code for the first $40 \mathrm{~s}$ into the RADYN simulations. The output spectra from the $\mathrm{RH}$ calculations (in units of $\mathrm{J} \mathrm{s}^{-1} \mathrm{~m}^{-2} \mathrm{~Hz}^{-1} \mathrm{sr}^{-1}$ ) were spectrally convolved to the IRIS instrumental Gaussian profile (50.54 $\mathrm{mA}$ in the near-UV [NUV] passband; De Pontieu et al. 2014), regridded into the IRIS spectral sampling, and converted into units of $\mathrm{DN} \mathrm{s}^{-1}$ pixel $^{-1}$, using a gain of $18 \mathrm{DN}_{\text {photon }^{-1}}$ for the NUV channel and effective area calculated as explained in Section 4.

The $\mathrm{Mg}$ II $h$ and $k$ lines are formed over a large range of atmospheric heights, and the interpretation of their spectra is not straightforward (e.g., Leenaarts et al. 2013a, 2013b; Pereira et al. 2013; Kerr et al. 2015, 2016). In the quiet Sun, they are usually observed as double peaked with a pronounced central reversal core. The modeling of these lines is also challenging, and the current models encounter difficulties in reproducing their observations in the AR plage or during flares (e.g., Carlsson et al. 2015; Rubio da Costa \& Kleint 2017). Here we aim to analyze the evolution of basic diagnostics of intensity and velocity as a function of our different nanoflare models, which can be directly compared with the IRIS observations, and postpone a more accurate modeling of the chromospheric emission and thorough analysis of the $\mathrm{Mg}$ II spectral features to a following work.

Figures 10 and 11 summarize the results of synthetic spectra over time for the $\mathrm{Mg}$ II $h \lambda 2803.529$ line, as a function of different heating models for the simulations with $15 \mathrm{Mm}$ halfloop length and initial apex temperatures of 1 and $3 \mathrm{MK}$, respectively. Similar results are found for the $\mathrm{Mg}$ II $k$ line and are thus not reported here. Different panels represent different nanoflare heating models, similarly to the Si IV spectra images shown in Section 4.1.

The Mg II $h$ line profile shows very peculiar features in the simulations with low-energy cutoff and TC for the loop with apex temperature of $1 \mathrm{MK}$ (panels (a) and (d) of Figure 10). For these runs, the spectra are in fact characterized by multipeak profiles with oppositely directed high-velocity Doppler shifts (up to $\approx \pm 40 \mathrm{~km} \mathrm{~s}^{-1}$ ), with the redshifted component being significantly more intense than the blueshifted counterpart. This redshifted component is the result of a strong downflow of cool plasma observed in the first $10 \mathrm{~s}$ of the simulation, which is caused by the large pressure gradient formed around $z \approx 1.7 \mathrm{Mm}$ (see Section 3.1). Such peculiar profiles and strong shifts have not been commonly seen in observations so far. If observed, they might represent an indication of heating by low-energy electrons or thermal conduction, as suggested by our models. However, it should be pointed out that in observations we are more likely observing a superposition of Mg II line profiles from the loops, as well as background chromospheric emission from the plage. In particular, a strong chromospheric heating may take place in the same AR (independently of the nanoflare heating) and determine the dominant emission for this line.

For simulations with high $E_{\mathrm{C}}(\geqslant 10 \mathrm{keV})$, the $\mathrm{Mg}$ II profile is mostly double peaked for the first $10 \mathrm{~s}$. The two peaks have a similar intensity, and the centrally reversed core is at rest. After $\approx 10 \mathrm{~s}$, the spectra are dominated by the red wing, and the line profile becomes almost single peaked.

For all the EB simulations in Figure 10 (panels (a)-(c)), we note an increase of intensity in the first $10 \mathrm{~s}$ as a quick response to the heating, similarly to what was observed in the Si IV synthetic spectra (Section 4.1).

In the TC simulation, the line reaches its peak intensity just after $10 \mathrm{~s}$ (see light curves in Figure 10(e)), because in this case the chromosphere is heated by a thermal conduction front from the corona, which has a longer timescale than the electron beam heating.

The synthetic spectra obtained for the denser $15 \mathrm{Mm}$ loop with apex temperature of $3 \mathrm{MK}$ (Figure 11) show a number of different features compared to the spectra in Figure 10 for a loop with apex temperature of $1 \mathrm{MK}$. First of all, the $\mathrm{Mg}$ II profile is always observed to be double peaked, and the increase of intensity of the line as a function of $E_{\mathrm{C}}$ is different compared to an almost empty loop. Specifically, for high $E_{\mathrm{C}}(\geqslant 10 \mathrm{keV})$, the intensity increase for a denser loop is larger than or comparable to the case of a lowdensity loop, whereas for the $5 \mathrm{keV}$ and TC simulations no significant $\mathrm{Mg}$ II brightening or dramatic change in the line profile is observed as a consequence of the nanoflare event. In the latter cases the chromosphere is not heated significantly, as most of the energy is dissipated in the corona and partly in the TR, before reaching the lower atmosphere. In addition, the line does not show strong shifts like those observed in the TC and $\mathrm{EB}, E_{\mathrm{C}}=5 \mathrm{keV}$ simulations of Figure 10. These differences are the result of the 1 MK loop being significantly less dense than the 3 MK loops. In a denser loop, the low-energy electrons are stopped at a higher height in the atmosphere and more energetic electrons are necessary to reach the chromosphere. 


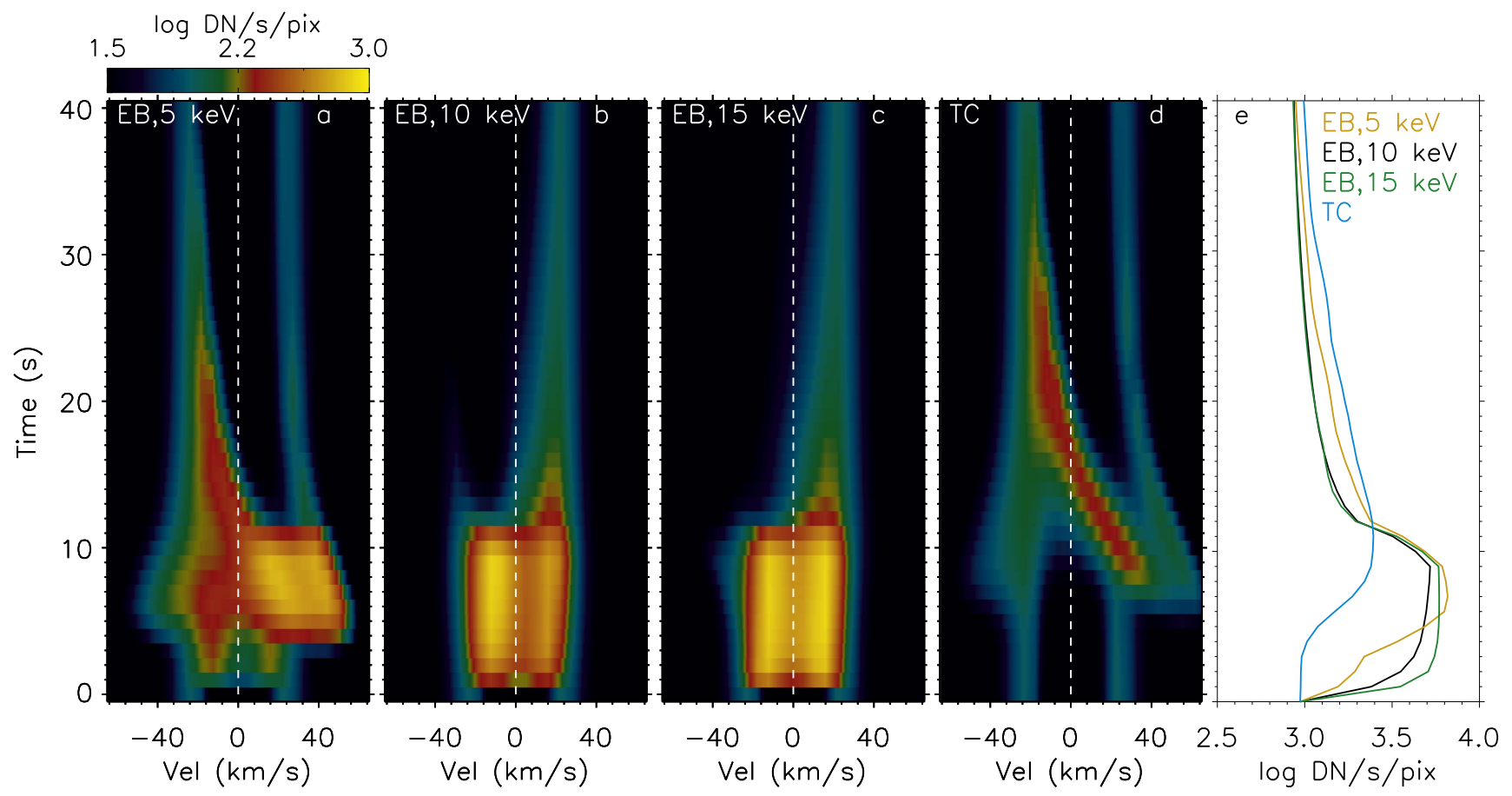

Figure 10. Synthetic Mg II $h$ spectra for the $15 \mathrm{Mm}$ loop, initial apex temperature of $1 \mathrm{MK}$, and different heating models (see the caption of Figure 5). The spectra are synthesized using the RH code with input atmosphere from the RADYN simulations, as explained in Section 5. Negative (positive) velocities indicate blueshifts (redshifts).

Finally, we note that the relative intensity increase of the $\mathrm{Mg}$ II line compared to the pre-flare conditions due to the nanoflare heating is not as dramatic as in the case of the $\mathrm{Si} I V$ line. The relative difference in the $\mathrm{Mg}$ II synthetic intensity between the $T_{\mathrm{LT}}=1$ and $3 \mathrm{MK}$ loops is also not as large as that observed for the Si IV synthetic spectra.

The results presented here show that the analysis of the $\mathrm{Mg}$ II lines can provide additional and different diagnostics of nanoflare heating from those provided by the TR Si IV spectra. For instance, the intensity increase of the $\mathrm{Mg}$ II due to the nanoflares is similar or stronger for the higher-density than low-density loops, in contrast to what is observed for the Si IV line. In addition, thermal conduction heating alone is not as efficient in increasing the $\mathrm{Mg}$ II intensity as the electron beam heating for either the low- or high-density loops. This is not true for the TR emission, where both thermal conduction and electron beam heating can produce a significant increase in the Si IV line intensity.

In this work, we have analyzed the general behavior of the $\mathrm{Mg}$ II lines to different nanoflare models in order to demonstrate their diagnostic capabilities. A thorough analysis of all their spectral features (also including the accurate modeling of the initial pre-nanoflare plage emission; see, e.g., Carlsson et al. 2015) is deferred to a future paper.

\section{Discussion: The Parameter Space}

In the previous sections, we discussed the impact of different heating properties and initial conditions of the loops on the atmospheric response and forward modeling of observables for our nanoflare simulations. In particular, we focused on discussing the results of varying the nonthermal electron energy distributions and investigating the difference between heating by electron beams and in situ heating of the corona, in loops with two different apex temperatures. In this section, we investigate in more detail the effect of modifying the initial physical conditions of the plasma, i.e., varying the length of the loops as well as their initial temperature. In addition, we briefly discuss the effect of assuming a different total energy release on the model predictions and observables.

\subsection{Other Loop Lengths}

We performed the same set of simulations presented in Section 3 for loops with $L / 2=50$ and $100 \mathrm{Mm}$, initial $T_{\mathrm{LT}}=1-3 \mathrm{MK}$, and corresponding loop-top electron number densities of $10^{8.0}-10^{9.0}$ (for the $50 \mathrm{Mm}$ loop) and $10^{7.1}-10^{8.6} \mathrm{~cm}^{-3}$ (for the $100 \mathrm{Mm}$ loop). We will discuss here only the main differences between the results of these runs and the ones presented in the previous sections.

The simulations with longer $L / 2=50$ and $100 \mathrm{Mm}$ and $T_{\mathrm{LT}}=1 \mathrm{MK}$ show very similar results to the same runs with a shorter loop length of $15 \mathrm{Mm}$ in the initial evolution of the nanoflare loop, whereas longer loops have a slower evolution on longer timescales (of the order of minutes). In particular, those runs present a similar behavior of atmospheric response as a function of low-energy cutoff, i.e., the smaller the electron energy, the more energy deposited directly in the corona. Consequently, similar trends of blueshifts versus redshifts over time are observed, with the main difference being the Doppler velocity values, which become progressively larger for longer loops. This is a consequence of the plasma being less dense in the longer loops, resulting in larger flows. The similar overall trend is caused by the fact that when the loop is almost empty, the electrons stream through the corona almost collisionlessly.

In contrast, the simulations with initial loop-top temperature of $3 \mathrm{MK}$ show more significant differences as a function of loop length. In particular, the intensity increase of the TR $\mathrm{Si}$ IV line as a response to the heating is larger in the $50 \mathrm{Mm}$ and $100 \mathrm{Mm}$ half-loop length simulations, as shown in 


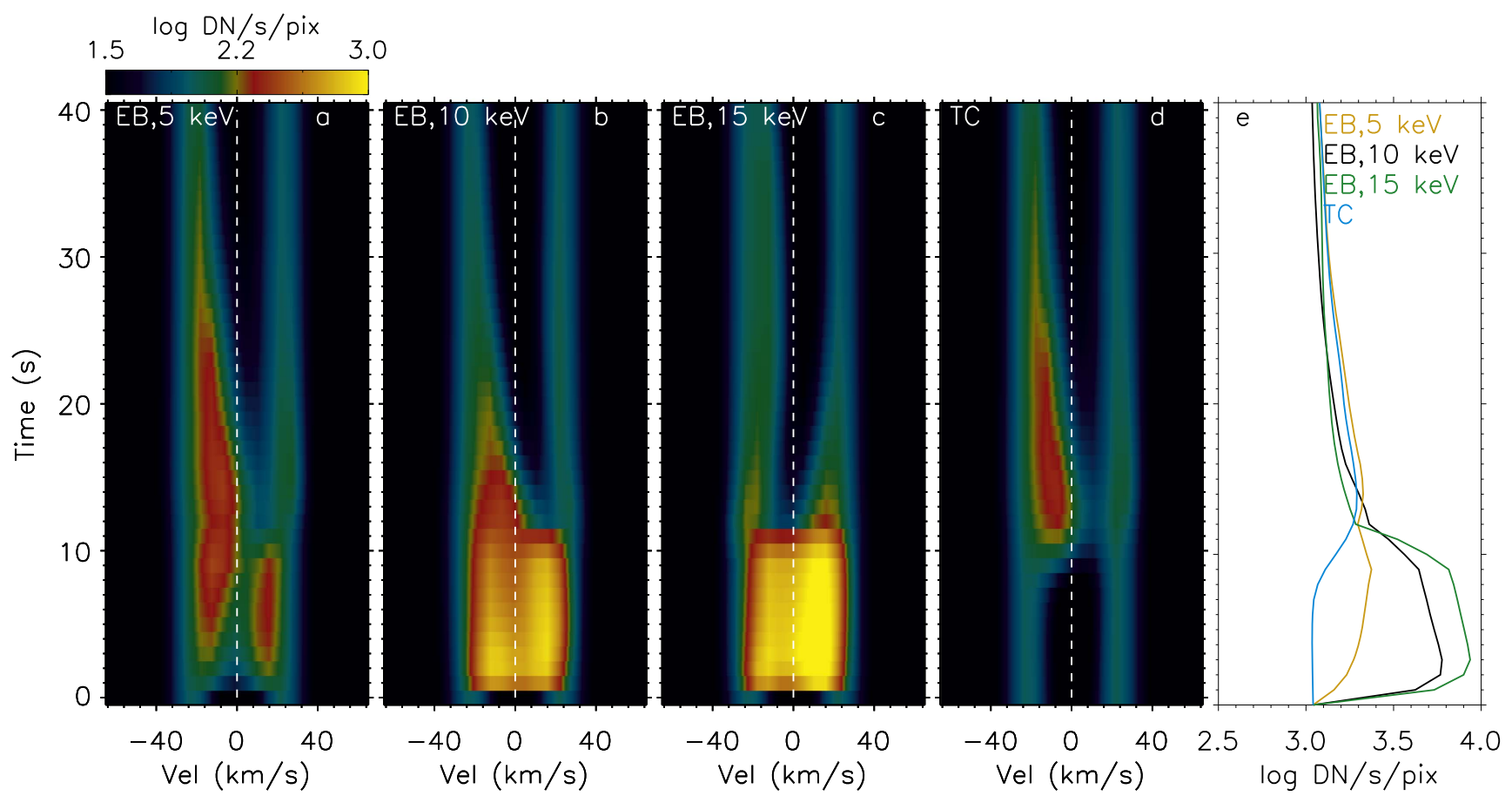

Figure 11. Synthetic Mg II $h$ spectra for the $15 \mathrm{Mm}$ loop, initial apex temperature of $3 \mathrm{MK}$, and different heating models. See the caption of Figure 10 for more details.

Figure 12 for the $100 \mathrm{Mm}$ loop. This is not surprising, as the lower density for these loops (compared to the $15 \mathrm{Mm}$ loop with the same initial temperature) causes the electrons to have less collisions in the corona and deposit more energy into the lower atmosphere. The trend of Doppler shifts versus electron energy and heating model is similar to the $15 \mathrm{Mm}$ case, with the only exception being that redshifts in the Si IV line are observed a bit later on $(\approx 20 \mathrm{~s})$ in the $\mathrm{EB} E_{\mathrm{C}}=5 \mathrm{keV}$ and $\mathrm{TC}$ simulations. This is due to the fact that, for longer loops, it takes longer for the plasma downflow and the thermal conduction front from the corona to reach the lower atmosphere. In addition, a small $\left(\approx 20 \mathrm{~km} \mathrm{~s}^{-1}\right)$ but very faint blueshift of the Si IV line is observed in the first $10 \mathrm{~s}$ of the $E_{\mathrm{C}}=5 \mathrm{keV}$ run for the $50 \mathrm{Mm}$ and $100 \mathrm{Mm}$ loops (here we only show the Si IV spectra for the $100 \mathrm{Mm}$ loop, as they are very similar). This initial blueshift is caused by the energy deposition of the electrons that are able to propagate through the corona and penetrate deeper down in the atmosphere. This is most likely not observed in the $15 \mathrm{Mm}$ half-loop simulations because in that case the plasma density is too high and a large part of the energy is dissipated in the corona without reaching the TR. Figure 12 also shows that the TC heating (panel (d)) does not affect the intensity of the Si IV line. This is likely caused by the fact that the loop is very long (more than 6 times longer than the $15 \mathrm{Mm}$ loop), and therefore most of the energy is dissipated along the coronal part of the loop without reaching the TR.

Another important difference between models with different loop lengths can be found in the synthetic AIA light curves, which differ both in their absolute values and in their time evolution. In particular, the $94 \AA$ filter emission is at least one order of magnitude lower in the 50 and $100 \mathrm{Mm}$ than in the $15 \mathrm{Mm}$ half-loop length simulations. This decrease in intensity is directly due to the plasma density being orders of magnitude lower in the two longer loops. In addition, the light-curve peaks occur later on in these simulations because of the slower thermal conduction timescale and the longer time it takes for the evaporating hot plasma to fill the longer loops.

Finally, although the details of the line profiles might change, the Mg II spectra show overall similar results in simulations for loops of different length, i.e., similar light curves over time and peak velocities. This is because the details of the coronal part of the loop do not strongly affect the results of the dense chromosphere.

\subsection{Loop-top Temperatures}

We run a series of simulations with both electron beam and in situ heating for $15 \mathrm{Mm}$ half-length loops with initial apex temperature of $5 \mathrm{MK}$ and electron number density of $\approx 10^{10.1} \mathrm{~cm}^{-3}$. These runs aim to reproduce a scenario where heating is applied to a nanoflare loop strand that has been previously heated to very large temperatures. Similarly to the case of loops with $T_{\mathrm{LT}}=3 \mathrm{MK}$, the electrons are mostly stopped in the corona, and very little energy can penetrate down to the TR and chromosphere. In addition, the in situ local heating in the thermal conduction simulations is mainly dissipated in the corona. As a result, no significant flows or intensity increase in the TR or chromospheric lines are observed. On the other hand, the synthetic AIA light curves are more intense (up to around one order of magnitude) than those obtained for the $T_{\mathrm{LT}}=1$ and $3 \mathrm{MK}$ runs, as can be best seen in Figure 13. The predicted increase of high-temperature emission in the $94 \AA$ filter is due to a combination of the initial coronal density being higher and the heating being mostly dissipated in the corona.

\subsection{Varying the Beam Energy Flux}

In order to investigate the impact of the nanoflare energy on the atmospheric response, we performed two additional sets of simulations assuming a total energy release of $10^{24}$ and $10^{25} \mathrm{erg}$ for the loop with $L / 2=15 \mathrm{Mm}$ and initial apex 


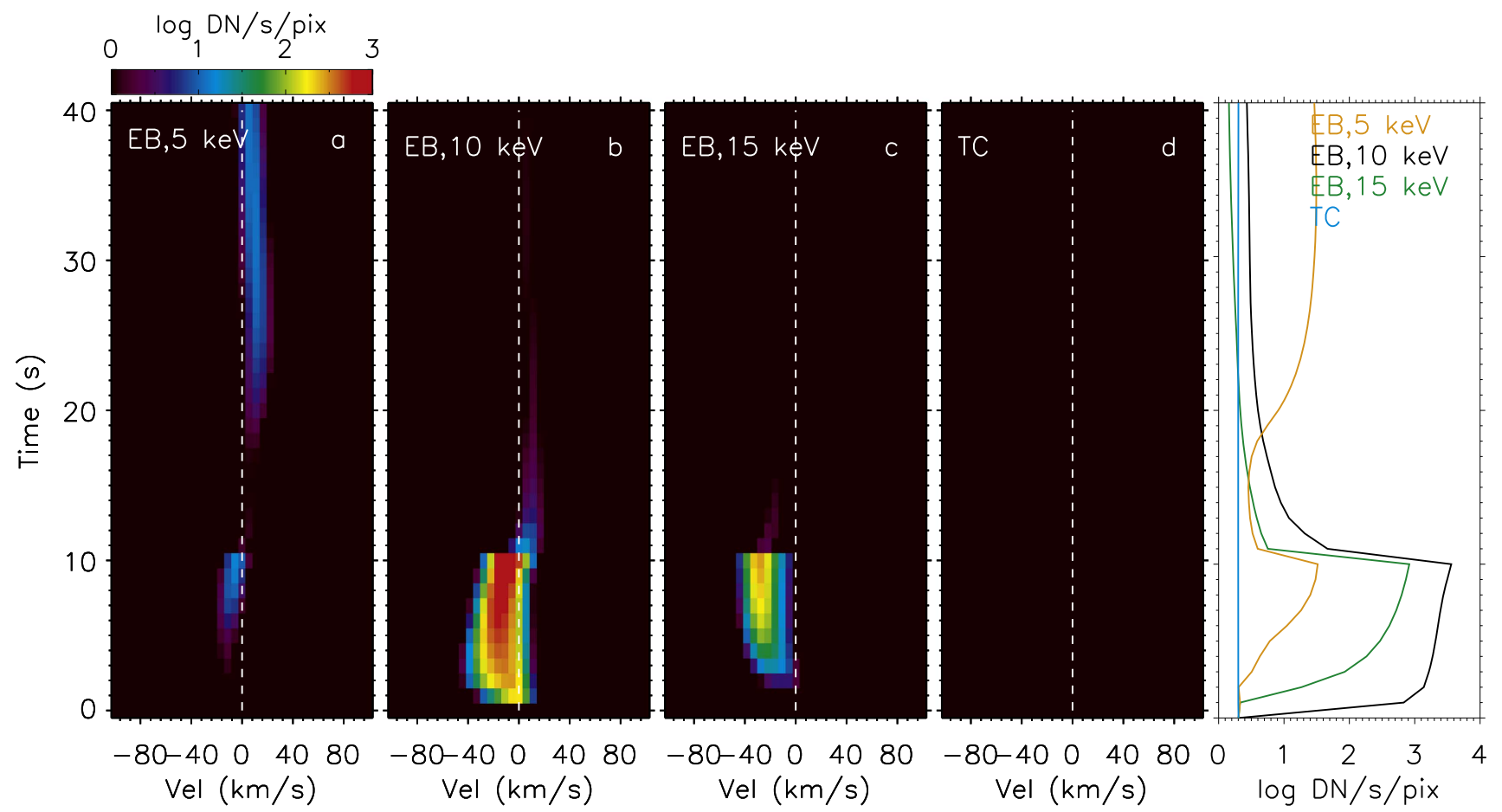

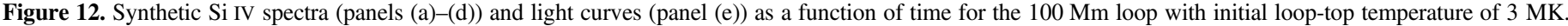
Negative (positive) velocities indicate blueshifts (redshifts). See caption of Figure 5 for more details.

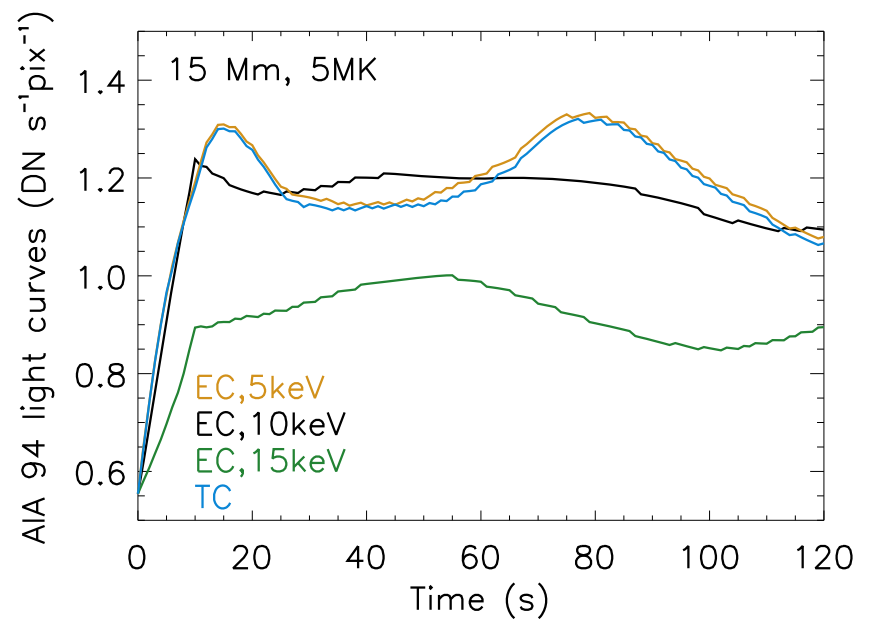

Figure 13. AIA synthetic light curves in the $94 \AA$ filter for our nanoflare models with $15 \mathrm{Mm}$ loop length and $T_{\mathrm{LT}}=5 \mathrm{MK}$. See caption of Figure 8 for more details.

temperature of $1 \mathrm{MK}$. These two values are representative of the range of energies that is typically assumed for nanoflaresize heating events and correspond (given the heating duration and loop cross section described in Section 2) to electron beam energy fluxes $F$ of $2 \times 10^{8}$ and $2 \times 10^{9} \mathrm{erg} \mathrm{cm}^{-2} \mathrm{~s}^{-1}$, respectively. Comparing electron distributions with the same spectral index and energy cutoff but different total energy fluxes corresponds to investigating the effect of varying the number of electrons in the beam at each energy. We also note that since the electron power laws that we assume are quite steep $(\delta=7)$, most of the electrons will have energy close to the cutoff value $E_{\mathrm{C}}$, and therefore they will initially deposit their energy at the same atmospheric height, regardless of the total energy flux.
The model runs that assume a total energy of $10^{25}$ erg show essentially the same qualitative results as the simulations presented in Section 3 for a nanoflare energy of $6 \times 10^{24} \mathrm{erg}$. In particular, they both show the same trend of up/downflows at different atmospheric layers as a function of electron energy cutoff. The main difference between the two numerical investigations is that in the first case $\left(E=10^{25} \mathrm{erg}\right)$ more energy is deposited in the atmosphere, causing faster plasma flows and a larger increase of density and emission measure for the optically thin plasma.

In contrast, the models assuming a smaller nanoflare energy $\left(10^{24} \mathrm{erg}\right)$ show somewhat different and interesting results. As mentioned before, the electrons will initially deposit their energy mostly in the same atmospheric layer as in the simulations with a higher total energy flux. However, the energy deposited there will be significantly lower for the $E=10^{24}$ erg than for the $6 \times 10^{24}$ or $10^{25}$ erg simulations. In the first case, the heating deposited is small enough that it can be more easily radiated away, avoiding the formation of a large overpressure region and the resulting strong increase of TR emission observed in the other two cases. In particular, the Si IV intensity for the $10^{24} \mathrm{erg}$ simulation will be significantly less intense than in the $6 \times 10^{24} \mathrm{erg}$ case. Another important difference between models with different total energy is in the atmospheric response, which in turn affects the evolution of the energy deposition over time. An interesting example is provided by the $\mathrm{EB}$ heating run with $E_{\mathrm{C}}=5 \mathrm{keV}$. We have shown in Section 3 that if the energy deposited by the $5 \mathrm{keV}$ electrons is high enough, a significant upflow of plasma will start filling the loops, and as a result the electrons streaming from the corona will be stopped at progressively higher heights. On the other hand, if the flows are slower (as in the $E=10^{24} \mathrm{erg}$ simulations), the electrons can deposit their energy deeper down in the atmosphere for a longer time. As a consequence, the large pressure gradient between the TR and 


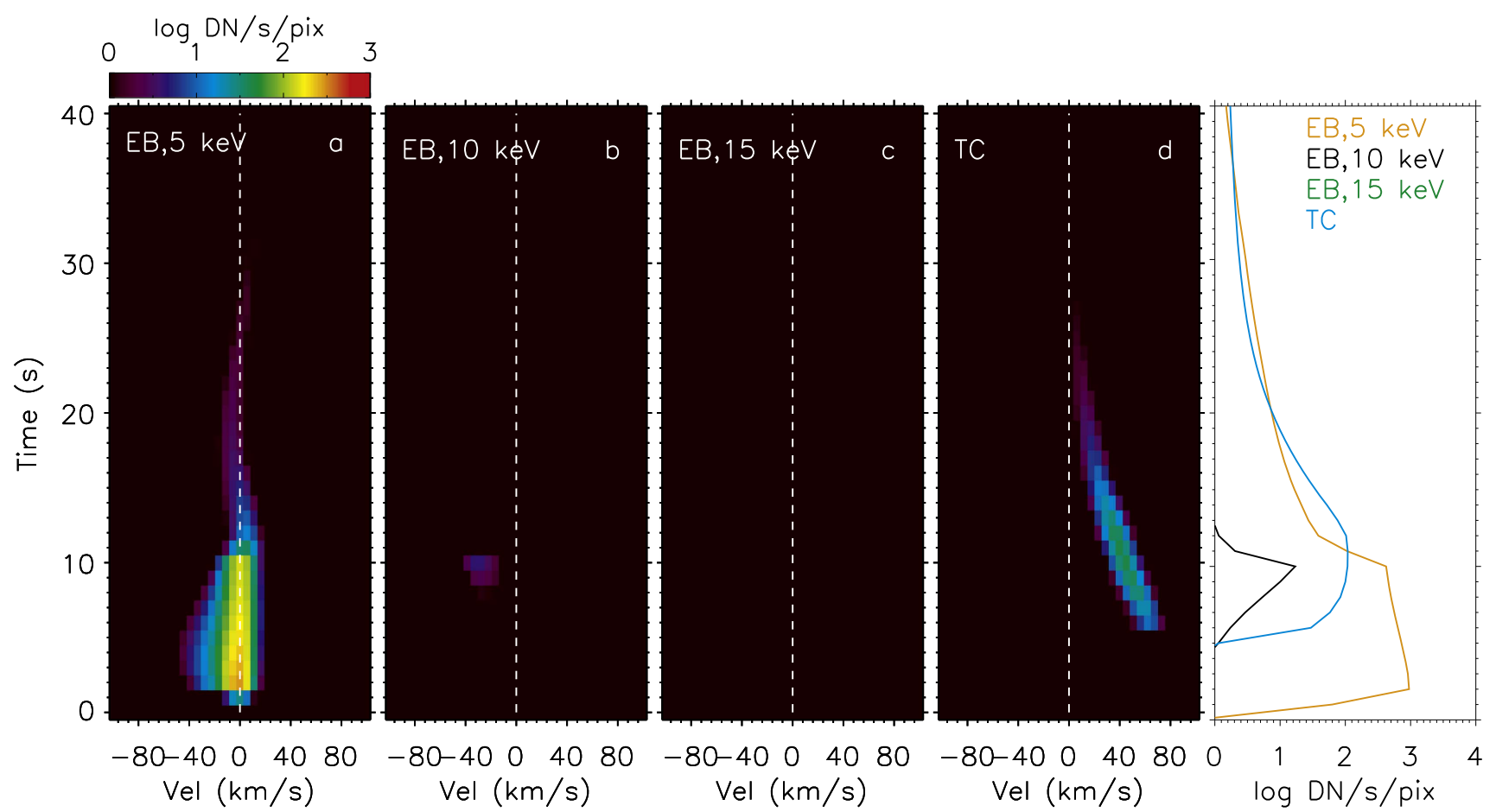

Figure 14. Synthetic Si IV spectra and light curves for the $15 \mathrm{Mm}$ loop with initial loop-top temperature of $1 \mathrm{MK}$ and total energy release of $E=10^{24}$ erg. Negative (positive) velocities indicate blueshifts (redshifts). See caption of Figure 5 for more details.

chromosphere, and therefore the strong downflow of Si IV plasma, will not be observed. In contrast, we find either no shifts or a blueshift $\left(20-30 \mathrm{~km} \mathrm{~s}^{-1}\right)$ in those spectra in the first few tens of seconds into the simulation, as can be best seen in Figure 14. This result suggests that a moderate blueshift in the Si IV line can also be found for electron energies as small as $5 \mathrm{keV}$, if the total energy of the beam is small enough or for certain physical conditions of the loop, as in the case of the loop with $L / 2=100 \mathrm{Mm}$ and $T_{\mathrm{LT}}=3 \mathrm{MK}$ presented in Section 6.2. However, it should be noted that although the $5 \mathrm{keV}$ case for a $10^{24} \mathrm{erg}$ nanoflare also produces a blueshift in the Si IV line, the main difference between this simulation and the ones with total energy of $6 \times 10^{24} \mathrm{erg}$ and larger $E_{\mathrm{C}}$ (10 and $15 \mathrm{keV}$ ) will be in the Si IV intensity, which is at least a factor of 10 larger for the latter simulations. Figure 14 also shows that the higher-energy electrons (with cutoffs of 10 and $15 \mathrm{keV}$ ) are not very efficient at heating the TR. This is because the energy dissipated in the chromosphere can be mostly radiated away without heating the TR and corona.

Finally, we note that the results of thermal conduction simulations do not change significantly as a function of total energy deposited during the nanoflare events. For instance, the TR Si IV spectra in the $E=10^{24} \mathrm{erg}$ simulation will still show a redshift, although on longer timescales than in the $E=6 \times 10^{24}$ or $10^{25} \mathrm{erg}$ simulations, as can be seen in Figure 14. We note that in all the cases we simulated, in situ heating of the corona cannot produce a blueshift in the Si IV line.

Figure 15 summarizes the results of Si IV velocity for the different models we have calculated here and discussed above. The figure shows the maximum Si IV centroid velocity attained at all times during each simulation as a function of the heating model (thermal conduction or electron beam heating with different electron energy cutoffs) for different initial conditions of the loop (length and temperature; top panel) and different total energies (bottom panel). The black symbols in parentheses indicate the cases where the line intensity was too low to be properly detected (below $10 \mathrm{DN}$ ).

The top panel of Figure 15 shows that there is an overall trend of Si IV centroid shifts as a function of heating models, where TC or EB simulations with low $E_{\mathrm{C}}$ present comparable redshifts (or sometimes larger for the TC simulations), while EB simulations tend to show larger blueshifts with increasing $E_{\mathrm{C}}$. The figure also indicates that simulations with different loop lengths and the same temperature provide very similar trends, especially for the $T_{\mathrm{LT}}=1 \mathrm{MK}$ simulations. We note that the initial density in these loops will be different, because their length is different, as a result of the way the loops are created in equilibrium conditions (as discussed in Section 2). However, the density difference is not as large as in loops with the same length but very different temperatures (i.e., 1 and $3 \mathrm{MK}$ loops). On the other hand, simulation runs with different initial loop temperatures (and very different initial densities) produce significantly different results for the TC and EB model with low $E_{\mathrm{C}}$. In addition, if the energy cutoff is large enough, simulations with different initial conditions for the loop produce approximately the same blueshift in the Si IV line.

The bottom panel of Figure 15 shows a similar trend of Doppler shifts as a function of heating model for total energies of $6 \times 10^{24}$ or $10^{25} \mathrm{erg}$. However, this seems not to be the case for simulations with significantly smaller energy (i.e., $10^{24} \mathrm{erg}$ ), where even energy distributions with low $E_{\mathrm{C}}$ can cause a small blueshift of the Si IV line, as also discussed previously.

\section{Conclusions}

We have carried out an extensive study of nanoflare-heated loops using RADYN 1D HD simulations and investigated the properties of the impulsive heating models and initial plasma conditions, within the parameter space described in Section 2. 

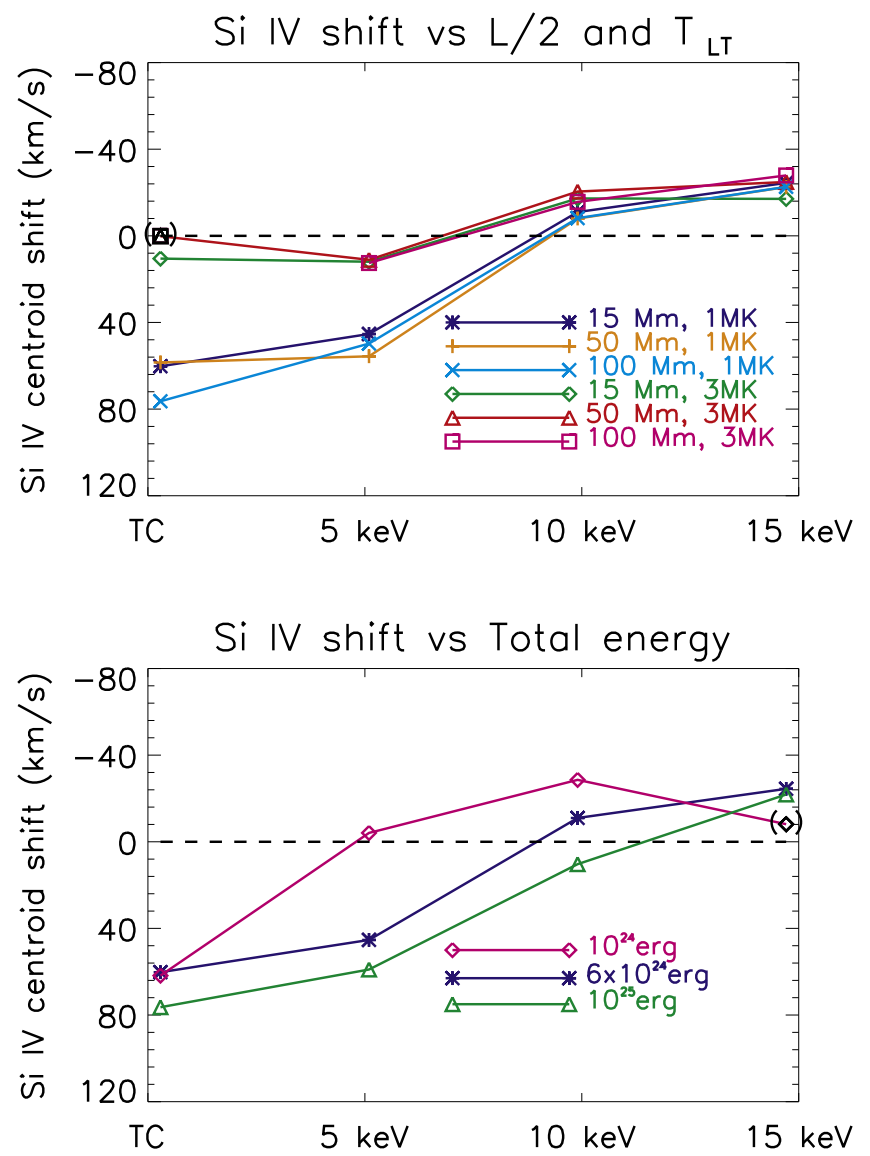

Figure 15. Maximum Si IV Doppler shift velocities obtained for each simulation as a function of different heating models (TC or EB with different values of $E_{\mathrm{C}}$ ). Top panel: different symbols and colors represent simulations with different initial conditions for the loops (half-loop lengths or temperature) as indicated in the legend. The black symbols in parentheses indicate when the line intensity was below $10 \mathrm{DN}$. Bottom panel: same as the top panel, but with different symbols indicating simulations using different total energies.

This study expands on the initial investigation presented and discussed in T14. Our aim was to reproduce a variety of possible physical scenarios that can be observed in nanoflare heating events, including different heat transport mechanisms (electron beam heating vs. in situ heating of the corona), as well as different physical initial conditions for the loops (empty vs. previously heated loop strands). We have chosen to simulate different heating models separately because this approach allows us to isolate the effects of energy deposition in the different cases. In reality, a combination of mechanisms (electron beams with different energies, as well as in situ heating deposition) is most likely to occur (see also discussion in Section 4.2).

For our simulations, we used the RADYN code, which provides an accurate modeling of the chromospheric emission in non-LTE and an advanced treatment of the accelerated electrons through the Fokker-Planck equations. We have also developed a more realistic initial atmospheric structure including a plage-like chromosphere, based on the work of Carlsson et al. (2015).

Our numerical investigation has provided several important results:

1. The atmospheric response changes significantly as a function of the electron energy cutoff, in agreement with the early findings of T14. In particular, lower-energy electrons tend to dissipate more energy in the corona, with results similar to the case when the corona is heated in situ. These electrons also produce very large upflows (a few hundred kilometers per second), in agreement with the prediction made by T14 and Reep et al. (2015) that also nanoflares with low-energy electrons could drive "explosive evaporation." In most cases we observe upflows from the TR, as the electrons do not penetrate as deep in the chromosphere as in the flare models, where more energetic electrons are typically assumed. Thermal conduction simulations are more effective at heating the loop and produce plasma up to $10-20 \mathrm{MK}$, which is the range of formation temperatures of highly ionized $\mathrm{Fe}$ atoms (such as Fe XXI-Fe XXIV). The emission of the upflowing hot plasma would, however, be very faint, because the coronal density is very low $\left(\approx 10^{8} \mathrm{~cm}^{-3}\right)$ and also the atoms may not be in ionization equilibrium (e.g., Reale \& Orlando 2008).

2. The initial conditions of the loops, i.e., temperature and density, are crucial in determining the response of the atmosphere and the IRIS and SDO/AIA observables. In particular, the same amount of energy deposited by the nanoflare will result in slower flows and a lower intensity of the TR emission for a dense loop (apex density and temperature of $\approx 10^{9.5} \mathrm{~cm}^{-3}$ and $3 \mathrm{MK}$, respectively). If the loop is even hotter and denser, for instance, a $5 \mathrm{MK}$ loop with apex density of $\approx 10^{10} \mathrm{~cm}^{-3}$, no significant heating of the TR has been obtained for the nanoflaresized events we simulated. Based on our simulations, we can therefore suggest that nanoflare-size heating events in loops with an apex density of more than $10^{9}-10^{9.5} \mathrm{~cm}^{-3}$ will not produce any significant TR footpoint brightenings for electron beam heating models with energy cutoff up to $15 \mathrm{keV}$ or thermal conduction models.

3. If the loop plasma has a low density, then the loop length is in many aspects not a crucial parameter: given the same input parameters for the electron beams and apex temperature, simulations with different loop lengths (and thus slightly different densities, but still low, below $10^{8} \mathrm{~cm}^{-3}$ for $50 \mathrm{Mm}$ and $100 \mathrm{Mm}$ loops) will produce qualitatively similar results overall, although the actual values of flows/intensity of the lines might vary. This does not apply to much denser loops: in this case, the length of the loop can determine different atmospheric responses and TR spectra for the same electron beam model, in particular for the low-energy electrons. This is because above a certain density $\left(\approx 10^{9.0} \mathrm{~cm}^{-3}\right)$ the electrons dissipate their energy more efficiently along the loop and the actual value of density might affect the way this occurs.

We synthesized the IRIS Si IV $1402.77 \AA$ and AIA $94 \AA$ emission, which can be directly compared with observations. As pointed out by T14, the analysis of the Si IV line and its evolution over time provide tight constraints on the possible heating scenarios. We have focused here on the detailed modeling of the optically thin plasma emission, whereas a thorough analysis and discussion of the chromospheric emission from our RADYN simulations is deferred to a future work.

Our key conclusions are summarized as follows: 
1. Our single nanoflare loop simulations, where the heating is released in $1 \mathrm{MK}$ and almost empty loops (with apex density of $\approx 10^{8.7} \mathrm{~cm}^{-3}$ ), show a sudden increase of Si IV emission (up to around 4 orders of magnitude) over a few seconds, in agreement with the observation of short-lived brightenings typical of the TR (e.g., Testa et al. 2013; T14). This applies to all the heating models in our numerical investigation, but the maximum Si IV intensity increase is obtained with the $10 \mathrm{keV}$ electrons, which are more efficient at heating the TR. For denser loops (with apex density of $\approx 10^{9.5} \mathrm{~cm}^{-3}$ ), more energetic electrons (10-15 keV) are needed to produce a rapid response of Si IV emission within the first few seconds of the simulation. For the model runs with $5 \mathrm{keV}$ electrons or in the case of in situ heating, the Si IV line becomes bright after only $10 \mathrm{~s}$, as a result of heating by a thermal conduction front from the corona. In any case, the predicted Si IV change in intensity is also much lower than in the case of a low-density loop strand, because the electrons are stopped more efficiently along a denser loop and consequently deposit less energy in the TR.

2. The observation of Si IV line shift provides a powerful diagnostic of the nanoflare heating properties. For empty loop strands, blueshifts are indicative of higher-energy electrons (10-15 keV), whereas simulations with lowenergy electrons or thermal conduction mostly show Si IV redshifts. Low-energy electrons and in situ heating of the corona give very similar results, and in most of the cases (especially for dense loops) it is virtually impossible to distinguish the two heating models based on the IRIS TR Si IV line or AIA coronal observables only. However, the threshold for TR blueshifts depends mainly on the total nanoflare energy, and even $5 \mathrm{keV}$ electrons can produce an Si IV blueshift in a $10^{24} \mathrm{erg}$ nanoflare. Combining the observation of Si IV Doppler shift and intensity can help constrain both the energy cutoff and energy of the nanoflare event. Finally, thermal conduction simulations can never produce TR plasma upflows (blueshifts), for all the cases we have analyzed in this work.

3. Lower-energy electrons are more efficient at heating the corona (as also pointed out by Reep et al. 2015; T14), resulting in more intense AIA $94 \mathrm{~A}$ emission. In particular, the AIA emission will be higher for loops with higher initial temperature and density in the corona. We also note that our single flare loops predict AIA $94 \AA$ intensities much lower than observed. This is not surprising, given that multistrand loop models, repeated heating events, and local energy release in the corona coexisting with accelerated particles might be needed to reproduce the observed coronal emission (e.g., Klimchuk 2006; Bradshaw et al. 2012; Reep et al. 2013). Nevertheless, our analysis provides an upper limit for the number of loop strands (of the order of hundreds) needed to match the observational results from AIA.

4. The chromospheric spectra show significantly different behavior compared with the TR emission. For high $E_{\mathrm{C}}$ $(\geqslant 10 \mathrm{keV})$ the $\mathrm{Mg}$ II intensity is larger for the denser atmosphere than for the initially low-density loop. For the low- $E_{\mathrm{C}}$ case, no significant increase in $\mathrm{Mg}$ II emission is observed for the dense loop, while more intense and highly redshifted Mg II lines, with complex spectral profiles, are predicted for the low-density case.

The results above suggest a possible scenario of nanoflare heating: (1) first, the intense and short-lived TR brightenings observed at times in the moss by IRIS and coronal imagers (e.g., Hi-C, AIA; e.g., Testa et al. 2013) can be explained by impulsive nanoflare heating in initially low-density loop strands (our $T_{\mathrm{LT}}=1 \mathrm{MK}$ loop model with apex density of $\approx 10^{8.7} \mathrm{~cm}^{-3}$ ); (2) subsequently, while the loops start being filled with high-temperature plasma and become denser (our $T_{\mathrm{LT}}=3 \mathrm{MK}$ loop model with apex density of $\approx 10^{9.6} \mathrm{~cm}^{-3}$ ), the electrons will no longer be effective at heating the TR plasma anymore, and therefore we will not see a significant increase of emission or shift in the Si IV spectra. On the other hand, the corona will still be heated to higher temperatures.

Our work has provided predictions for a large sample of plausible nanoflare heating scenarios, which can be directly compared with the IRIS diagnostics of variable footpoint emission associated with coronal heating events in ARs. The findings presented here have also allowed for a better understanding of the heating properties as a function of different input parameters in nanoflare-size events.

Finally, we note that the possibility of heating by Alfvén wave dissipation in flares has been recently investigated numerically by Reep \& Russell (2016) and Kerr et al. (2016). Further work will be dedicated to including the Alfvén wave heating mechanism in our grid of simulations and comparing the predictions from this model with the ones obtained by the electron beam and in situ heating mechanisms that we have presented in this work.

We thank the referee for the useful comments that helped improve the paper. V.P. and P.T. are supported by NASA grant NNX15AF50G and by contract 8100002705 from LockheedMartin to SAO. P.T. also acknowledges support by NASA grant NNX15AF47G and contract NNM07AB07C to SAO. B.D.P. is supported by NNG09FA40C (IRIS). This research was supported by the Research Council of Norway through its Centres of Excellence scheme, project no. 262622, and through grants of computing time from the Programme for Supercomputing. Resources supporting this work were provided by the NASA High-End Computing (HEC) Program through the NASA Advanced Supercomputing (NAS) Division at Ames Research Center. IRIS is a NASA small explorer mission developed and operated by LMSAL, with mission operations executed at NASA Ames Research center and major contributions to downlink communications funded by ESA and the Norwegian Space Centre. CHIANTI is a collaborative project involving researchers at the universities of Cambridge (UK), George Mason, and Michigan (USA). The authors thank the International Space Science Institute (ISSI) for their support and hospitality during the meetings of the ISSI team "New Diagnostics of Particle Acceleration in Solar Coronal Nanoflares from Chromospheric Observations and Modeling."

\section{ORCID iDs}

V. Polito (1) https://orcid.org/0000-0002-4980-7126

P. Testa (1) https://orcid.org/0000-0002-0405-0668

J. Allred (1) https://orcid.org/0000-0003-4227-6809

B. De Pontieu (1) https://orcid.org/0000-0002-8370-952X

M. Carlsson (1) https://orcid.org/0000-0001-9218-3139 
T. M. D. Pereira (1) https://orcid.org/0000-0003-4747-4329

Milan Gošić (ib https://orcid.org/0000-0002-5879-4371

Fabio Reale (i) https://orcid.org/0000-0002-1820-4824

\section{References}

Allred, J. C., Kowalski, A. F., \& Carlsson, M. 2015, ApJ, 809, 104 Antiochos, S. K., Karpen, J. T., DeLuca, E. E., Golub, L., \& Hamilton, P. 2003, ApJ, 590, 547

Berger, T. E., de Pontieu, B., Fletcher, L., et al. 1999, SoPh, 190, 409

Boerner, P. F., Testa, P., Warren, H., Weber, M. A., \& Schrijver, C. J. 2014, SoPh, 289, 2377

Bradshaw, S. J., Klimchuk, J. A., \& Reep, J. W. 2012, ApJ, 758, 53

Brooks, D. H., \& Warren, H. P. 2009, ApJL, 703, L10

Carlsson, M., Leenaarts, J., \& De Pontieu, B. 2015, ApJL, 809, L30

Carlsson, M., \& Stein, R. F. 1992, ApJL, 397, L59

Carlsson, M., \& Stein, R. F. 1995, ApJL, 440, L29

Carlsson, M., \& Stein, R. F. 1997, ApJ, 481, 500

Culhane, J. L., Harra, L. K., James, A. M., et al. 2007, SoPh, 243, 19

De Pontieu, B., Berger, T. E., Schrijver, C. J., \& Title, A. M. 1999, SoPh, 190, 419

De Pontieu, B., De Moortel, I., Martinez-Sykora, J., \& McIntosh, S. W. 2017, ApJL, 845, L18

De Pontieu, B., Erdélyi, R., \& de Wijn, A. G. 2003, ApJL, 595, L63

De Pontieu, B., McIntosh, S. W., Carlsson, M., et al. 2011, Sci, 331, 55

De Pontieu, B., Title, A. M., Lemen, J. R., et al. 2014, SoPh, 289, 2733

Del Zanna, G. 2012, A\&A, 546, A97

Del Zanna, G. 2013, A\&A, 558, A73

Del Zanna, G., Dere, K. P., Young, P. R., Landi, E., \& Mason, H. E. 2015, A\&A, 582, A56

Del Zanna, G., O’Dwyer, B., \& Mason, H. E. 2011, A\&A, 535, A46

Dere, K. P., Landi, E., Mason, H. E., Monsignori Fossi, B. C., \& Young, P. R. 1997, A\&AS, 125, 149

Dorfi, E. A., \& Drury, L. O. 1987, JCoPh, 69, 175

Feldman, U., Mandelbaum, P., Seely, J. F., Doschek, G. A., \& Gursky, H. 1992, ApJS, 81, 387

Fletcher, L., \& De Pontieu, B. 1999, ApJL, 520, L135

Freeland, S. L., \& Handy, B. N. 1998, SoPh, 182, 497

Gustafsson, B. 1973, UppAn, 5, 6

Handy, B. N., Acton, L. W., Kankelborg, C. C., et al. 1999, SoPh, 187, 229

Hannah, I. G., Hudson, H. S., Battaglia, M., et al. 2011, SSRv, 159, 263

Harrison, F. A., Craig, W. W., Christensen, F. E., et al. 2013, ApJ, 770, 103

Judge, P. G., Paraschiv, A., Lacatus, D., Donea, A., \& Lindsey, C. 2017, ApJ, 838, 138

Kerr, G. S., Fletcher, L., Russell, A. J. B., \& Allred, J. C. 2016, ApJ, 827, 101

Kerr, G. S., Simões, P. J. A., Qiu, J., \& Fletcher, L. 2015, A\&A, 582, A50

Klimchuk, J. A. 2006, SoPh, 234, 41

Klimchuk, J. A. 2015, RSPTA, 373, 20140256

Kobayashi, K., Cirtain, J., Winebarger, A. R., et al. 2014, SoPh, 289, 4393

Landi, E., Young, P. R., Dere, K. P., Del Zanna, G., \& Mason, H. E. 2013, ApJ, 763, 86

Leenaarts, J., Pereira, T. M. D., Carlsson, M., Uitenbroek, H., \& De Pontieu, B. 2013a, ApJ, 772, 89
Leenaarts, J., Pereira, T. M. D., Carlsson, M., Uitenbroek, H., \& De Pontieu, B. 2013b, ApJ, 772, 90

Lemen, J. R., Title, A. M., Akin, D. J., et al. 2012, SoPh, 275, 17

Martens, P. C. H., Kankelborg, C. C., \& Berger, T. E. 2000, ApJ, 537, 471

Martínez-Sykora, J., De Pontieu, B., Testa, P., \& Hansteen, V. 2011, ApJ, 743,23

Mikić, Z., Lionello, R., Mok, Y., Linker, J. A., \& Winebarger, A. R. 2013, ApJ, 773, 94

Müller, D. A. N., Hansteen, V. H., \& Peter, H. 2003, A\&A, 411, 605

Müller, D. A. N., Peter, H., \& Hansteen, V. H. 2004, A\&A, 424, 289

O’Dwyer, B., Del Zanna, G., Mason, H. E., Weber, M. A., \& Tripathi, D. 2010, A\&A, 521, A21

Parker, E. N. 1988, ApJ, 330, 474

Pereira, T. M. D., Leenaarts, J., De Pontieu, B., Carlsson, M., \& Uitenbroek, H. 2013, ApJ, 778, 143

Pereira, T. M. D., \& Uitenbroek, H. 2015, A\&A, 574, A3

Peres, G., Reale, F., \& Golub, L. 1994, ApJ, 422, 412

Peres, G., Reale, F., Serio, S., \& Pallavicini, R. 1987, ApJ, 312, 895

Pesnell, W. D., Thompson, B. J., \& Chamberlin, P. C. 2012, SoPh, 275, 3

Peter, H., Bingert, S., Klimchuk, J. A., et al. 2013, A\&A, 556, A104

Priest, E. R., Heyvaerts, J. F., \& Title, A. M. 2002, ApJ, 576, 533

Reale, F. 2014, LRSP, 11, 4

Reale, F. 2016, ApJL, 826, L20

Reale, F., Guarrasi, M., Testa, P., et al. 2011, ApJL, 736, L16

Reale, F., Landi, E., \& Orlando, S. 2012, ApJ, 746, 18

Reale, F., \& Orlando, S. 2008, ApJ, 684, 715

Reale, F., Testa, P., Klimchuk, J. A., \& Parenti, S. 2009, ApJ, 698, 756

Reep, J. W., Bradshaw, S. J., \& Alexander, D. 2015, ApJ, 808, 177

Reep, J. W., Bradshaw, S. J., \& Klimchuk, J. A. 2013, ApJ, 764, 193

Reep, J. W., \& Russell, A. J. B. 2016, ApJL, 818, L20

Rubio da Costa, F., \& Kleint, L. 2017, ApJ, 842, 82

Saint-Hilaire, P., Krucker, S., Christe, S., \& Lin, R. P. 2009, ApJ, 696, 941

Tajfirouze, E., Reale, F., Peres, G., \& Testa, P. 2016, ApJL, 817, L11

Testa, P., De Pontieu, B., Allred, J., et al. 2014, Sci, 346, 1255724

Testa, P., De Pontieu, B., \& Hansteen, V. 2016, ApJ, 827, 99

Testa, P., De Pontieu, B., Martínez-Sykora, J., et al. 2013, ApJL, 770, L1

Testa, P., Drake, J. J., \& Landi, E. 2012, ApJ, 745, 111

Testa, P., Peres, G., \& Reale, F. 2005, ApJ, 622, 695

Testa, P., \& Reale, F. 2012, ApJL, 750, L10

Testa, P., Reale, F., Landi, E., DeLuca, E. E., \& Kashyap, V. 2011, ApJ, 728,30

Testa, P., Saar, S. H., \& Drake, J. J. 2015, RSPTA, 373, 20140259

Tripathi, D., Mason, H. E., Del Zanna, G., \& Young, P. R. 2010, A\&A, $518, \mathrm{~A} 42$

Ugarte-Urra, I., \& Warren, H. P. 2014, ApJ, 783, 12

Uitenbroek, H. 2001, ApJ, 557, 389

van Ballegooijen, A. A., Asgari-Targhi, M., Cranmer, S. R., \& DeLuca, E. E. 2011, ApJ, 736, 3

Warren, H. P., Winebarger, A. R., \& Brooks, D. H. 2010, ApJ, 711, 228

Warren, H. P., Winebarger, A. R., Mariska, J. T., Doschek, G. A., \& Hara, H. 2008, ApJ, 677, 1395

Winebarger, A. R., Schmelz, J. T., Warren, H. P., Saar, S. H., \& Kashyap, V. L. 2011, ApJ, 740, 2 